\title{
Finitely axiomatizable strongly minimal groups
}

\author{
Thomas Blossier and Elisabeth Bouscaren
}

February 28, 2007

\begin{abstract}
We show that if $G$ is a strongly minimal finitely axiomatizable group, the division ring of quasi-endomorphisms of $G$ must be an infinite finitely presented ring.
\end{abstract}

Questions about finite axiomatizability of first order theories are nearly as old as model theory itself and seem at first glance to have a fairly syntactical flavor. But it was in order to show that totally categorical theories cannot be finitely axiomatized that, in the early eighties, Boris Zilber started developing what is now known as "Geometric stability theory". Indeed, as is often the case, in order to answer such a question, one needs to develop a fine analysis of the structure of models in the class involved and to understand exactly how each model is constructed.

The easiest way to force a structure to be infinite by one first order sentence is to impose an ordering without end points, or a dense ordering, thus making the structure unstable. It was hence rather natural to wonder about theories at the other extremity of the stability spectrum, and in the early 60's the question was posed whether there existed finitely axiomatizable totally categorical theories or simply uncountably categorical theories [21], [16].

Each model of a totally categorical theory is prime over a strongly minimal set. It is not too difficult to see that a totally categorical strongly minimal set cannot be finitely axiomatizable ([14]). Much more complicated, the proof of the non finite axiomatizability for the whole class goes through a characterization of the geometries associated to totally categorical strongly minimal sets (locally modular and locally finite) and then an analysis of how any model is "built" around the strongly minimal set ([22], [23] and [6] where the result is proved for all $\omega$-stable $\omega$-categorical theories).

Around the same time as Zilber's negative answer for the totally categorical case, Peretjat'kin produced an example of a finitely axiomatized $\aleph_{1}$-categorical theory [18]. This example was in the following years simplified by Baisalov (see [9, 12.2, Example 5]). This final example has Morley Rank equal to 2, thus still leaving open the question of the existence of a finitely axiomatizable strongly minimal set (Morley rank and degree equal to 1). Furthermore all the known examples of finitely axiomatizable $\aleph_{1}$-categorical theories are rather similar and constructed around a strongly minimal set with trivial 
pregeometry, also leaving open the question of the existence of a finitely axiomatizable $\aleph_{1}$-categorical theory with non trivial pregeometry.

In 1994, Hrushovski ([10]) showed that any finitely axiomatizable $\aleph_{1}$-categorical theory must have locally modular pregeometry, thus reducing the remaining open questions to two very different cases:

- the existence of a finitely axiomatizable trivial strongly minimal set

- the existence of any finitely axiomatizable $\aleph_{1}$-categorical theory which contains a locally modular strongly minimal group.

The canonical example of a strongly minimal locally modular non trivial theory is that of infinite $K$-vector spaces, for a fixed division ring $K$. It is open whether there exists any finitely axiomatizable complete theory of $R$-modules, for $R$ any ring, but it is very easy to check that if $K$ is an infinite division ring which is finitely presented as a ring, then the theory of $K$-vector spaces can be finitely axiomatized. Unfortunately, the existence of such a division ring is open (see section 3). Conversely, it was originally shown by Paljutin ([17]), in a paper where he characterizes finitely axiomatizable uncountably categorical quasi-varieties, that, if the theory of infinite $K$-vector spaces is finitely axiomatizable, then $K$ is finitely presented as a ring (see section 3.2).

In the paper cited above, Hrushovski conjectures that, more generally, a finitely axiomatizable $\aleph_{1}$-categorical non trivial theory exists if and only if such an infinite finitely presented division ring exists. Any $\aleph_{1}$-categorical non trivial locally modular theory must contain a locally modular strongly minimal group $G$, and the geometry associated to such a group is that of infinite $K$-vector spaces, where $K$ is the division ring of quasi-endomorphisms of $G$ (see section 2 for the definitions). The precise conjecture in $[10]$ is that, in any finitely axiomatizable $\aleph_{1}$-categorical non trivial theory, the associated division ring of quasi-endomorphisms is infinite and finitely presented as a ring.

One should remark that although every $\aleph_{1}$-categorical non trivial locally modular theory must contain a definable strongly minimal group, one cannot use general arguments to transfer down the finite axiomatizability to the strongly minimal group. We will see in section 3.1 some general assumptions under which finite axiomatizability can be transferred (bi-interpretability, definable finite partition). But, it is not even true in general that, if $M$ is finitely axiomatizable and contained in the algebraic closure of a strongly minimal set $D$ ( $M$ is then said to be almost strongly minimal), the strongly minimal set $D$, with the induced structure from $M$, must be finitely axiomatizable. In the finitely axiomatized $\aleph_{1}$-categorical Morley rank 2 theory which was mentioned above, for example, the whole structure $M$ is contained in the algebraic closure of a strongly minimal subset $D \subset M \times M$ (the diagonal), whose induced structure is that of the integers with the successor function, which is not finitely axiomatizable.

One last remark, in past years, work around strongly minimal finitely axiomatizable trivial sets has also centered around a conjecture relating their existence to the existence of an infinite group with specific properties (see section 3 for some further details).

In this paper we show that Hrushovski's conjecture holds for strongly minimal groups, and more generally for Morley Rank one groups: If $G$ is a finitely axiomatizable strongly minimal group, then the division ring of quasi-endomorphisms of $G$ must be infinite and 
finitely presented (Theorem 3.19).

By Hrushovski's result, we know that such a group must be locally modular. This enables us to reduce to the case when $G$ is a strongly minimal Abelian structure. Then we show (Proposition 3.16) that if $G$ is a finitely axiomatizable strongly minimal abelian structure, the division ring $K$ of quasi-endomorphisms of $G$ must be infinite and that the theory of $K$-vector spaces must also be finitely axiomatizable (Lemma 3.15).

We begin in section 1 by recalling or proving some general facts about abelian structures, under the precise form they will be needed later. In particular, we describe, in section 1.3, the theory which will end up being both finitely axiomatizable and interdefinable with the theory of $K$-vector spaces. In section 2 we recall the basic facts about the ring of quasi-endomorphisms of a locally modular strongly minimal group and we look at strongly minimal abelian structures. In section 3 , we consider the question of finite axiomatization. We begin by a somewhat technical section (3.1) where we give precise definitions of finite axiomatizability in the case of infinite languages and we show how this notion transfers when changing languages or structures. In order to be as selfcontained as possible on the subject of finite axiomatizability, in section 3.2, we recall very precisely the two classical examples (regular group actions and vector spaces). In the next section (section 3.3), we prove the main theorem, that if a strongly minimal abelian structure is finitely axiomatizable, then its division ring of quasi-endomorphisms, $K$, must be infinite and the theory of $K$-vector spaces must be finitely axiomatizable. Finally in the last section (3.4) we conclude for strongly minimal groups and more generally for groups of Morley Rank one.

We would like to thank the many people with whom we have had very helpful discussions since we started getting interested in questions of finite axiomatizability, in particular, Ehud Hrushovski, Alexandre Ivanov, David Lippel, Dugald MacPherson, Mike Prest and Gabriel Sabbagh.

\section{Abelian structures}

\subsection{Axiomatization and quantifier elimination}

In this first section, we recall the precise statements about axiomatization and $p p$ elimination of quantifiers for abelian structures

We define an abelian structure $\mathbb{G}$, to be a commutative group

$$
\mathbb{G}=<G,+,-, 0,\left(H_{i}\right)_{i \in I}>,
$$

where each $H_{i}$ is a subgroup of some $G^{n_{i}}$. We denote by $L_{0}$ the language $\left\{+,-, 0,\left(H_{i}\right)_{i \in I}\right\}$. We are going to consider expansions of abelian structures by constants and we will denote by $L_{c}$ the language of an expansion of $\mathbb{G}$ by some constants in a subset $C$, i.e. $L_{c}=L_{0} \cup\{c: c \in C\}$.

Recall that the set of positive primitive formulas is the closure of the atomic formulas by conjunction and existential quantifiers. 
It has been well-known for years that in a complete theory of modules, every formula is equivalent to a Boolean combination of $p p$-formulas and that a complete theory of modules is axiomatized by so-called invariant statements describing the index of pairs of positive primitive definable subgroups ([3], [20]).

The similar result for abelian structures has also been known for a long time (abelian structures were originally introduced by E. Fisher in [7]) but was never published in any "official" form until it appeared as a special case in the general treatment of theories given by cosets in [8]. As we are dealing with questions of finite axiomatization, it is important for us to be extremely precise about the form of the axioms and the language we are working in. For this reason we will recall briefly the precise definitions we need and state, mostly without proofs, the results under the exact form we require. Some similar considerations appear also in [5].

Lemma 1.1. Let $\phi\left(x_{1}, \ldots, x_{n}\right)$ be a consistant positive primitive formula in $L_{0}$ in $n$ variables $(n \geq 1)$ and without parameters. Then $\left\{a \in G^{n}: \mathbb{G} \models \phi(a)\right\}$ is a subgroup of $G^{n}$.

Note that the set of $p p$-definable subgroups in $\mathbb{G}$ corresponds to the closure of the groups $\left(H_{i}: i \in I\right)$, the trivial groups $(\{0\}$ and $G)$, the diagonal of $G^{2}$ and the graph of the addition by, cartesian product, permutation of coordinates, intersection and projection.

Lemma 1.2. Let $\phi(\bar{x}, \overline{0})$ be a pp-formula from $L_{0}$ without parameters, which defines a subgroup in $G^{n}$. Let $\bar{d}$ be a tuple from $G$. Then $\phi(\bar{x}, \bar{d})$ is empty or is a coset of the pp-definable subgroup defined by $\phi(\bar{x}, \overline{0})$.

A $p p$-formula in the language $L_{c}$ is equivalent to $\phi(\bar{x}, \bar{c})$ where $\phi$ is a $p p$-formula from $L_{0}$ and $\bar{c}$ is a tuple of constants. In particular a subgroup of $G^{n}$ which is definable by a $p p$-formula from $L_{c}$ is in fact already $p p$-definable in $L_{0}$ without parameters.

Let $T(\mathbb{G})$ be the following set of sentences from $L_{0}$ :

- $G$ is a commutative group,

- for each original predicate $H_{i}$ from $L_{0}, H_{i}$ is a subgroup of $G^{n_{i}}$,

- the equivalence sentences: all sentences of the form $\forall \bar{x}(\phi(\bar{x}) \leftrightarrow \psi(\bar{x}))$, for $\phi$ and $\psi$ pp-formula which define the same subgroup of $G^{n}$ (note that these sentences give the following relations between $p p$-definable subgroups : inclusion, intersection, projection and equality up to a permutation of variables),

- the dimension sentences: for each pair $H \subset H^{\prime}$ of $p p$-definable subgroups of $G$, such that the index of $H$ in $H^{\prime}$ is equal to $n$, the sentence " $\left[H^{\prime}: H\right]=n$ "; for each pair $H \subset H^{\prime}$ of $p p$-definable subgroups of $G$, such that the index of $H$ in $H^{\prime}$ is infinite in $G$, the infinite scheme of sentences " $\left[H^{\prime}: H\right] \geq k$ ", for every $k \geq 1$.

Fact 1.3. The theory $T(\mathbb{G})$ is complete in the language $L_{0}$ and admits quantifier elimination to the pp-formulas, that is, every formula is equivalent modulo $T(\mathbb{G})$ to a Boolean combination of pp-formulas.

Note that it follows easily that every abelian structure is stable. 
Corollary 1.4. The theory of $\mathbb{G}$ in the language $L_{c}$ is given by $T(\mathbb{G})$ together with the pp-type of the constants (i.e. for each pp-definable group $H$, we have to describe the $H$-congruences on the set of constants).

Corollary 1.5. Let $a \in G$ and $B \subset G$, then $a$ is algebraic on $B$ in the $L_{c}$-structure $\mathbb{G}$ if and only if $a$ is in a $B$-definable coset of some finite pp-definable subgroup of $G$.

Proof. By elimination to $p p$-formulas, the type of $a$ over $B$ is given by the set $X$ of $B$-definable cosets of $p p$-definable subgroups to which $a$ belongs, and the set $Y$ of $B$ definable cosets of pp-definable subgroups to which $a$ does not belong. Note that $X$ is closed under finite intersections. By compactness, as $a$ is algebraic over $B$, there is some coset $A$ in $X$ and some cosets $B_{1}, B_{2}, \ldots, B_{n}$ from $Y$ such that $A \backslash\left(B_{1} \cup \ldots \cup B_{n}\right)$ is finite non empty. We can suppose that each $B_{i}$ is contained in $A$, by taking its intersection with $A$. Then $A=a+H=\left\{a_{1}\right\} \cup \ldots \cup\left\{a_{m}\right\} \cup\left(d_{1}+H_{1}\right) \cup \ldots \cup\left(d_{n}+H_{n}\right)$, where $\left\{a_{i}\right\}$ is considered as a coset of the trivial group, $d_{i}+H_{i}=B_{i}$ and $H_{i} \subset H$. By Neumann's Lemma, if some coset $a+H$ is covered by a finite number of cosets, then it is covered by those cosets which correspond to subgroups of finite index in $H$. It follows that $A$ itself is finite.

We finish with a remark that will be very useful in the sequel:

Proposition 1.6. Let $\mathbb{G}$ be an abelian structure in $L_{c}$. Let $H \subset G^{n}$ be any definable connected subgroup (with parameters). Then $H$ is pp-definable. In particular, $H$ is definable over $\emptyset$.

Proof. Let $H$ be a connected definable subgroup of $G^{n}$. By $p p$-elimination, there is a set $A$ such that $H$ is equivalent to a Boolean combination of $p p$-formulas with parameters in $A$. We can suppose that the unique generic type of $H, q$, is defined and stationary over $A$. As $q$ is a complete type over $A$, we can suppose that there are $\bar{a}_{0}, \bar{a}_{1}, \ldots, \bar{a}_{m} \in A$ and $p p$-definable (over $\emptyset$ ) subgroups $\phi_{0}, \phi_{1}, \ldots, \phi_{m}$ of $G^{n}$ such that:

1. for each $i>0, \phi_{i}$ is a subgroup of $\phi_{0}$,

2. $\left(\bar{a}_{0}+\phi_{0}\right) \backslash\left(\bar{a}_{1}+\phi_{1} \cup \ldots \cup \bar{a}_{m}+\phi_{m}\right) \subseteq H$,

3. every generic of $H$ over $A$ is in $\left(\bar{a}_{0}+\phi_{0}\right) \backslash\left(\bar{a}_{1}+\phi_{1} \cup \ldots \cup \bar{a}_{m}+\phi_{m}\right)$,

4. for each $i>0$, each $\phi_{i}$ is of infinite index in $\phi_{0}$ : indeed if $\phi_{i}$ has finite index in $\phi_{0}$, by enlarging $A$ if necessary, we can suppose that $\bar{a}_{0}+\phi_{0}=\bigcup_{1 \leq j \leq k} \bar{e}_{j}+\phi_{i}$, with $\bar{e}_{j} \in A$, and replace $\bar{a}_{0}+\phi_{0}$ with one of the $\bar{e}_{j}+\phi_{i}$.

It follows that $H=\phi_{0}$ : the difference of two generics of $H$ (over $A$ ) is in $\phi_{0}$, so $H \subset \phi_{0}$. Conversely, let $h$ be a generic of $H$ over $A$. Take a generic $x$ of $\phi_{0}$ over $A h$. Since for each $i>0, \phi_{i}$ is a subgroup of $\phi_{0}$ of infinite index, $x \notin\left(\bar{a}_{i}-h\right)+\phi_{i}$. Thus $x+h \in\left(\bar{a}_{0}+\phi_{0}\right) \backslash\left(\bar{a}_{1}+\phi_{1} \cup \ldots \cup \bar{a}_{m}+\phi_{m}\right)$ and $x \in H$. 


\subsection{Direct sums}

The following direct sum construction plays an essential role in the paper.

Let $\mathbb{S}$ be an abelian structure in the language $L_{0}$, and let $\mathbb{G}$ be an abelian structure in the language $L_{c}$. Let $\mathbb{G}_{S}:=\mathbb{G} \oplus \mathbb{S}$, be the $L_{c}$-structure with universe the group $G_{S}=G \oplus S$ and with the obvious interpretation of the symbols in the language: each constant $c$ is interpreted by $(c, 0)$; for $H$ of arity $n$, we interpret $H$ in $G_{S}$ by $H(G) \oplus H(S)$ where $H(G):=\left\{(h, 0) \in G_{S}{ }^{n}: \mathbb{G}=H(h)\right\}$ and $H(S):=\left\{(0, h) \in G_{S}{ }^{n}: \mathbb{S} \models H(h)\right\}$. If $g=\left(g_{1}, \ldots, g_{n}\right) \in G^{n}$ and $s=\left(s_{1}, \ldots, s_{n}\right)$, we will use both notations $g+s$ or $(g, s)$ to denote the element $\left(g_{1}+s_{1}, \ldots, g_{n}+s_{n}\right) \in G_{S}{ }^{n}$.

Lemma 1.7. For each pp-formula $\phi(x)\left(x=\left(x_{1}, \ldots, x_{n}\right)\right)$, from $L_{0}, \phi\left(G_{S}\right)=\phi(G) \oplus$ $\phi(S)$, i.e., for $h_{1} \in G$, and $h_{2} \in G_{S}, \mathbb{G}_{S} \models \phi\left(h_{1}+h_{2}\right)$ if and only if $\mathbb{G} \models \phi\left(h_{1}\right)$ and $\mathbb{S} \models \phi\left(h_{2}\right)$.

Proof. By induction on $p p$-formulas. For atomic formulas, it follows from the way $\mathbb{G}_{S}$ is defined as being the direct sum of $\mathbb{G}$ and $\mathbb{S}$ as $L_{0}$-structures. For a conjunction of two $p p$ formulas, it follows easily from the fact that $G_{S}$ is the direct sum of $G$ and $S$ as groups. There remains to check the case of a projection. Let $\phi\left(x_{1}, \ldots, x_{n}\right)$ be a $p p$-formula such that $\phi\left(G_{S}\right)=\phi(G) \oplus \phi(S)$ and consider the $p p$-formula $\psi:=\exists x_{1} \phi$. We have trivially that $\psi(G) \oplus \psi(S) \subset \psi\left(G_{S}\right)$. Let $\left(a_{2}, \ldots, a_{n}\right) \in \psi\left(G_{S}\right)$. Then there is $a_{1} \in G_{S}$ such that $\left(a_{1}, \ldots, a_{n}\right) \in \phi\left(G_{S}\right)=\phi(G) \oplus \phi(S)$. So $\left(a_{1}, \ldots, a_{n}\right)=\left(b_{1}, \ldots, b_{n}\right)+\left(c_{1}, \ldots, c_{n}\right)$, with $\left(b_{1}, \ldots, b_{n}\right) \in \phi(G)$ and $\left(c_{1}, . ., c_{n}\right) \in \phi(S)$. But then $\left(a_{2}, \ldots, a_{n}\right)=\left(b_{2}, \ldots, b_{n}\right)+\left(c_{2}, \ldots, c_{n}\right)$ with $\left(b_{2}, \ldots, b_{n}\right) \in \psi(G)$ and $\left(c_{2}, . ., c_{n}\right) \in \psi(S)$.

Remark. In particular, if $\phi(\bar{x}, \bar{y})$ is a $p p$-formula in $L_{0}$ and $\bar{c}=c_{1}, \ldots, c_{n}$ are some constants from $C$. Then $\mathbb{G}_{S} \models \phi(g+s, c)$ if and only if $\mathbb{G} \models \phi(g, c)$ and $\mathbb{S} \models \phi(s, 0)$.

\subsection{The totally transcendental case}

We consider an abelian structure $\mathbb{G}$ with constants, in the language $L_{c}$, and we denote its theory by $\mathbb{T}$.

Recall that in a totally transcendental group, there is no infinite strictly decreasing sequence of definable subgroups. It follows easily that the same is true for cosets:

Lemma 1.8. If $M$ is a totally transcendental group then every infinite intersection of definable cosets in $M^{n}$ is equivalent to a finite intersection.

The following lemmas (1.9 to 1.12) are well known for the case of modules (in the usual language for modules, see [20]) and mostly folklore for abelian structures. The proofs are similar to the ones in the case of modules.

Definition. Let $\mathbb{M}$ be a model of $\mathbb{T}$. We say that $\mathbb{M}$ is pure injective if every set of $p p$-formulas with parameters in $M$ (with possibly infinitely many variables) which is finitely realized in $M$ is realized in $M$.

Lemma 1.9. If $\mathbb{T}$ is totally transcendental then every model of $\mathbb{T}$ is pure injective. 
Proposition 1.10. If $\mathbb{G}_{0}$ is an elementary substructure of $\mathbb{G}$ and is pure injective, then there exists $f$, an $L_{c}$-homomorphism from $G$ to $G_{0}$, such that $f$ is the identity on $G_{0}$.

Corollary 1.11. If $\mathbb{G}_{0}$ is an elementary substructure of $\mathbb{G}$, then $G=G_{0} \oplus G_{1}$ and for each pp-definable subgroup $H \subset G^{n}$ in $L_{0}, H=H \cap\left(G_{0}{ }^{n}\right) \oplus H \cap\left(G_{1}{ }^{n}\right)$.

Proof. Let $G_{1}=\operatorname{ker} f$ where $f$ is given by the previous proposition. Then $g=f(g)+$ $(g-f(g))$ and $g-f(g) \in K e r f$ for each $g \in G$ : indeed $f(g-f(g))=f(g)-f(f(g))=$ $f(g)-f(g)=0$. We have the same property for cartesian product. So we can suppose that $H \subset G$ to simplify the notation. Since $H$ is a $p p$-definable subgroup and $f$ is an $L_{0}$-homomorphism, $f(H) \subset H \cap G_{0}$ and so $f(H)=H \cap G_{0}$. Thus $H=H \cap G_{0} \oplus H \cap G_{1}$ : for each $h \in H, f(h) \in H \cap G_{0}$ and $(h-f(h)) \in \operatorname{ker} f \cap H$.

Let $G=G_{0} \oplus G_{1}$, as above, where $\mathbb{G}_{0}$ is an elementary substructure of $\mathbb{G}$, and $G_{1}=\operatorname{ker} f, f$ given by Prop. 1.10. Let $\mathbb{G}_{1}$ be the following abelian structure on $G_{1}$ in the language $L_{0}$ :

$$
\mathbb{G}_{1}=<G_{1},+,-, 0,\left(H_{i} \cap G_{1}^{n_{i}}\right)_{i \in I}>\text {. }
$$

Then $\mathbb{G}=\mathbb{G}_{0} \oplus \mathbb{G}_{1}$ as abelian structures (see section 1.2) and by lemma 1.7:

Lemma 1.12. For every subgroup $H$ of $G^{n}$ definable by a pp-formula $\phi$ in $\mathbb{G}$, the group $\phi\left(G_{1}\right):=\left\{a \in G_{1}{ }^{n}: \mathbb{G}_{1} \models \phi(a)\right\}$ is equal to $H \cap G_{1}^{n}$.

Let $\mathbb{T}_{1}(\mathbb{G})$ be the following modification of the axioms $T(\mathbb{G})$ :

- the axioms for abelian groups,

- for each original predicate $H$ from $L_{0}$, " $H$ is a subgroup",

- the equivalence sentences from $T(\mathbb{G})$,

- "[ $[\phi: \psi]$ is infinite" for every pair of $p p$-formulas such that in $\mathbb{G}, \psi(G) \subset \phi(G) \subset G$ and $[\phi(G): \psi(G)]$ is infinite,

- $[\phi: \psi]=1$ for every pair of $p p$-formulas such that in $\mathbb{G}, \psi(G) \subset \phi(G) \subset G$ and $[\phi(G): \psi(G)]$ is finite.

Proposition 1.13. If $\mathbb{G}$ is $\left|G_{0}\right|^{+}$-saturated, $\mathbb{T}_{1}(\mathbb{G})$ axiomatizes the complete theory of the abelian structure $\mathbb{G}_{1}$, that is $\mathbb{T}_{1}(\mathbb{G}) \vdash T\left(\mathbb{G}_{1}\right)$.

Proof. We show first that $\mathbb{G}_{1}$ is a model of $\mathbb{T}_{1}:=\mathbb{T}_{1}(\mathbb{G})$. By Lemma 1.12, $\mathbb{G}_{1}$ satisfies the equivalence sentences from $T(\mathbb{G})$. Let $K \subset H$ be a pair of $p p$-definable subgroups of $G$. If $[H: K]$ is infinite then by $\left|G_{0}\right|^{+}$-saturation of $\mathbb{G},[H: K]>\left|G_{0}\right|$. Thus $\left[H \cap G_{1}: K \cap G_{1}\right]$ is infinite. If $[H: K]$ is equal to $k$, since $G_{0}$ is an elementary substructure, there are $a_{1}, \ldots, a_{k} \in G_{0}$ such that $H=a_{1}+K \cup \ldots \cup a_{k}+K$. Let $x \in H \cap G_{1}$. Then $x=a_{i}+b$ where $b \in K$. Let $f$ be as in Prop. 1.10, then $0=f(x)=f\left(a_{i}\right)+f(b)=a_{i}+f(b)$. But $f(b) \in K$, so $x \in K$ and $H \cap G_{1}=K \cap G_{1}$.

Now we show that $T\left(\mathbb{G}_{1}\right)$ is a consequence of $\mathbb{T}_{1}$. Let $\phi$ and $\psi$ be a pair of $p p$-formulas from $L_{0}$ which define subgroups $H$ and $K$ of $G^{n}$ such that $H \cap G_{1}^{n}=K \cap G_{1}^{n}$. We have to show that the sentence $\forall \bar{x}(\phi(\bar{x}) \leftrightarrow \psi(\bar{x}))$ is a consequence of $\mathbb{T}_{1}$. Note first that if $K \subset H$ and $[H: K]$ is finite, this follows from the last axioms by an easy induction on 
$n$. But we can consider the pair $K \cap H \subset H$ and remark that $[H: K \cap H]$ is finite: indeed, if $[H: K \cap H]$ was infinite, then $\left[H \cap G_{1}^{n}: K \cap H \cap G_{1}^{n}\right]$ would be infinite. For the dimension sentences, suppose now that $H$ and $K$ are $p p$-definable subgroups of $G$ such that $K \cap G_{1} \subset H \cap G_{1}$. Then the sentence for the index of $\left[H \cap G_{1}: K \cap G_{1}\right]$ is a consequence of the sentence from $\mathbb{T}_{1}$ corresponding to the pair of $p p$-formulas $\psi \cap \phi$ and $\phi$.

Remark 1.14. Note that $G_{1}$ contains no non trivial finite $p p$-definable subgroup. So, in $\mathbb{G}_{1}$, the algebraic closure of the empty set is reduced to 0 and algebraic closure corresponds to definable closure.

Suppose now that $\mathbb{G}$ is a model of $\mathbb{T}$ and that $\mathbb{S}$ is a model of the theory $\mathbb{T}_{1}:=\mathbb{T}_{1}(\mathbb{G})$, and consider the $L_{c}$-structure $\mathbb{G}_{S}=\mathbb{G} \oplus \mathbb{S}$ as in section 1.2 , then:

Corollary 1.15. If $\mathbb{S}$ is a model of $\mathbb{T}_{1}$ and $\mathbb{G}$ is a model of $\mathbb{T}$, then $\mathbb{G}_{S}$ is a model of $\mathbb{T}$ and the map $i$ from $G$ into $G_{S}, i(g)=(g, 0)$ is an $L_{c}$-elementary isomorphism (i.e. $G \oplus\{0\}$ is an $L_{c}$-elementary substructure of $\left.\mathbb{G}_{S}\right)$.

Proof. Let $\phi$ and $\psi$ be two $p p$-formulas such that $\phi(G) \subset \psi(G)$. Then $\phi(S) \subset \psi(S)$ (by equivalence sentences). If $[\psi(G): \phi(G)]=k$ then $\psi(S)=\phi(S)\left(\right.$ by $\left.\mathbb{T}_{1}\right)$, so $\left[\psi\left(G_{S}\right)\right.$ : $\left.\phi\left(G_{S}\right)\right]=k$. If $[\psi(G): \phi(G)]=\infty$ then $\left[\psi\left(G_{S}\right): \phi\left(G_{S}\right)\right]=\infty$. Thus, $\mathbb{G}_{S}$ is a model of $\mathbb{T}$, so $G \oplus\{0\}$ and $\mathbb{G}_{S}$ are elementarily equivalent, and it follows by $p p$-elimination in $\mathbb{T}$ and Lemma 1.7 that $G \oplus\{0\} \prec \mathbb{G}_{S}$.

\section{The strongly minimal case}

We remind the reader that a structure $M$ is said to be strongly minimal if every definable subset of $M$ (with parameters possibly in an elementary extension of $M$ ) is finite or co-finite. If $M$ is strongly minimal, model theoretic algebraic closure (denoted acl) defines a pregeometry on $M$, in particular, for any $X \subset M$, the dimension of $X$ (the cardinality of a maximal algebraically free subset in $X$ ) is well defined. We say that $M$ is trivial, or has trivial pregeometry if, for all $A \subset M, \operatorname{acl}(A)=\bigcup_{a \in A} a c l\{a\}$. We say that $M$ is locally modular if for all algebraically closed $X, Y \subset M$, such that $\operatorname{dim}(\operatorname{acl}(X \cap Y))>0, \operatorname{dim}(X \cup Y)=\operatorname{dim}(X)+\operatorname{dim}(Y)-\operatorname{dim}(X \cup Y)$. We will explicitly state the results we use about locally modular strongly minimal groups. For proofs and details we refer to [19], [4] or [15].

By a strongly minimal group, we mean, as usual, that $G$ is a group with possibly extra structure, in a language $L$, which is strongly minimal as an $L$-structure.

Let $\mathcal{G}=(G, L)$ be a strongly minimal group in a language $L$. Let $G_{0}:=G \cap \operatorname{acl}(\emptyset)$. A quasi-endomorphism of $\mathcal{G}$ is a connected subgroup $H$ of $G^{2}$, definable over $a c l^{e q}(\emptyset)$, different from $G^{2}$, such that the first projection of $H$ is equal to $G$. It follows that $H$ is strongly minimal. We define the kernel of $H$ and the cokernel of $H$ to be respectively:

$$
\operatorname{Ker} H:=\{a \in G:(a, 0) \in H\}, \operatorname{Coker}(H):=\{a \in G:(0, a) \in H\} .
$$

The cokernel of $H$ is always finite, and if $H$ is not trivial, that is if $H \neq G \times 0$, the kernel of $H$ is finite. We denote by $Q S(\mathcal{G})$ the set of quasi-endomorphisms of $\mathcal{G}$. 
Remark: By strong minimality, if $G_{0}$ is infinite, $G_{0}$ is an elementary substructure of $\mathcal{G}$ and in that case, all quasi-endomorphisms of $\mathcal{G}$ are actually definable over $G_{0}$. In any case, if $\mathcal{M}_{0}$ is a prime model for $T h(\mathcal{G})$, all quasi-endomorphisms are definable over $M_{0}$.

A quasi-endomorphism induces an endomorphism of $G / G_{0}$ : if $H \in Q S(\mathcal{G})$, then $\{(a+$ $\left.\left.G_{0}, b+G_{0}\right):(a, b) \in H\right\}$ is the graph of an endomorphism $f_{H}$ of $G / G_{0}$. Furthermore the map which to every $H \in Q S(\mathcal{G})$ assigns the endomorphism $f_{H}$ is a bijection from $Q S(\mathcal{G})$ onto the ring of the "quasi-definable" endomorphisms of $G / G_{0}$. The ring of endomorphisms of $G / G_{0}$ induces the structure of a division ring on $Q S(\mathcal{G})$.

In the case of a locally modular group, the pregeometry on $\mathcal{G}$ defined by the relation of algebraic closure corresponds to the geometry of $Q S(\mathcal{G})$-vector spaces. More precisely:

Fact 2.1. Let $\mathcal{G}$ be a locally modular strongly minimal group, let $b, a_{1}, \ldots, a_{n} \in G$. then $b \in \operatorname{acl}\left(a_{1}, \ldots, a_{n}\right)$ if and only if there are quasi-endomorphisms $S_{1}, \ldots, S_{n}$ and elements $h_{1}, \ldots, h_{n} \in G$ such that for each $i, 1 \leq i \leq n$,

$$
\left(a_{i}, h_{i}\right) \in S_{i} \text { and } b-\left(h_{1}+\ldots+h_{n}\right) \in G_{0} .
$$

Any strongly minimal abelian structure $\mathbb{A}$ is locally modular and by 1.6 , all quasiendomorphisms of $\mathbb{A}$ are definable over $\emptyset$.

By general results about one-based groups (see section 3.4), any locally modular strongly minimal group is "almost interdefinable" with a strongly minimal abelian structure. This will enable us at the end to reduce to the case of finitely axiomatizable abelian structures.

From now on in this section, $\mathbb{G}$ is a strongly minimal abelian structure with constants, in the language $L_{c}$.

Consider, $T(\mathbb{G})$, the axiomatization of the theory of $\mathbb{G}$ given in the previous sections, and $\mathbb{T}_{1}=\mathbb{T}_{1}(\mathbb{G})$, the associated theory.

Note that one can see directly from the axiomatizations of the form $T(\mathbb{G})$ when an abelian structure is strongly minimal: by pp-elimination $\mathbb{G}$ will be strongly minimal if and only if $\mathbb{G}$ is infinite and for any pp-definable subgroup $H$ of $G, H$ is finite or equal to $G$.

Lemma 2.2. The theory $\mathbb{T}_{1}$ is strongly minimal.

Proof. First a model of $\mathbb{T}_{1}$ must be infinite (the formula $x=x$ is a pp-formula). Let $\phi$ be a pp-definable subgroup of $G$. By strong minimality of $\mathbb{G}$, either $\phi$ is finite, or $\phi$ is equal to $G$. Hence in any model $\mathbb{H}$ of $\mathbb{T}_{1}, \phi$ is trivial or $\phi=H$. By $p p$-elimination, $\mathbb{T}_{1}$ is also strongly minimal.

We also know (see Remark 1.14) that in any model of $\mathbb{T}_{1}$, acl $(\emptyset)=\{0\}$ and acl $=d c l$. It follows easily that if $\mathbb{G}_{1}$ is a model of $\mathbb{T}_{1}$ and if $K_{1}$ is the division ring of quasiendomorphisms of $\mathbb{G}_{1}$, the structure on $\mathbb{G}_{1}$ is exactly the $K_{1}$-vector space structure. But we want to check that $K_{1}=K$, where $K$ is the division ring of quasi-endomorphisms of $\mathbb{G}$.

Let us recall the definition of interdefinability: 
Definition 2.3. Let $\mathcal{M}_{1}=\left(M, L_{1}\right)$ and $\mathcal{M}_{2}=\left(M, L_{2}\right)$ be, respectively, $L_{1}$ and $L_{2^{-}}$ structures with the same universe $M$. Let $A \subset M$, we say that $\mathcal{M}_{1}$ is $A$-definable in $\mathcal{M}_{2}$ if every $A$-definable subset in $\mathcal{M}_{1}$ is $A$-definable in $\mathcal{M}_{2}$, equivalently, if every symbol in the language $L_{1}$ is $A$-definable in $\mathcal{M}_{2}$. We say that $\mathcal{M}_{1}$ and $\mathcal{M}_{2}$ are $A$-interdefinable if each $\mathcal{M}_{i}$ is $A$-definable in the other, equivalently, if $\mathcal{M}_{1}$ and $\mathcal{M}_{2}$ have the same $A$-definable subsets.

Proposition 2.4. Let $K$ be the division ring of quasi-endomorphisms of $\mathbb{G}$. Then, if $\mathbb{S}$ is any model of $\mathbb{T}_{1}, \mathbb{S}$ carries a $K$-vector space structure with which it is $\emptyset$-interdefinable.

Proof. Let $\mathbb{G}_{S}^{\prime}=\mathbb{G}^{\prime} \oplus \mathbb{S}$, where $\mathbb{G}^{\prime}$ is a countable elementary substructure of $\mathbb{G}$ (take for $G^{\prime}, G_{0}=\operatorname{acl}(\emptyset)$, if it is infinite) and $\mathbb{S}$ is any model of $\mathbb{T}_{1}$. Then $\mathbb{G}_{S}^{\prime}$ is a model of $\mathbb{T}$, the theory of $\mathbb{G}(1.15)$. Let $\phi_{r}(x, y)$ be a $p p$-formula which defines the quasiendomorphism $r \in K$. By the axioms in $\mathbb{T}_{1}$, in $S \times S, \phi_{r}(x, y)$ defines a subgroup such that its first projection is equal to $S$, which is strict and connected, hence which is a quasi-endomorphism of $S$. The kernel, $\left\{y \in S: S=\phi_{r}(0, y)\right\}$, must be trivial, as well as the co-kernel. So $\phi_{r}(x, y)$ defines an endomorphism of $S$. It follows easily that the set of $p p$-formulas $\left(\phi_{r}: r \in K\right)$ induce a $K$-vector space structure on $S$.

Now, it suffices to prove that any two tuples, $a_{1}, \ldots, a_{n}$ and $b_{1}, \ldots, b_{n}$, of $S$ have the same $L_{0}$-type if they have the same $K$-vector space type.

Claim. Let $a, b, a_{1}, . ., a_{n} \in S$ be such that $a$ is $K$-linearly independent from $a_{1}, \ldots, a_{n}$ and $b$ is also $K$-linearly independent from $a_{1}, \ldots, a_{n}$, then there exist an $L_{0}$-automorphism of $S$ which sends $a$ to $b$ and fixes $a_{1}, \ldots, a_{n}$.

Proof. In the structure $\mathbb{G}_{S}^{\prime},(0, a) \notin \operatorname{acl}\left(\left(G^{\prime} \oplus\{0\}\right) \cup\left\{\left(0, a_{1}\right), \ldots,\left(0, a_{n}\right)\right\}\right)$ (in the language $\left.L_{c}\right)$ : otherwise, there would be $r_{1}, \ldots, r_{n} \in K,\left(x_{1}, y_{1}\right), \ldots,\left(x_{n}, y_{n}\right) \in G_{S}^{\prime}$ and $g \in G^{\prime}$ such that

$$
(0, a)=\left(x_{1}, y_{1}\right)+\ldots+\left(x_{n}, y_{n}\right)+(g, 0) \text { and } G_{S}^{\prime} \models \phi_{r_{i}}\left(\left(0, a_{i}\right),\left(x_{i}, y_{i}\right)\right) .
$$

But then, $a=y_{1}+\ldots+y_{n}=r_{1} a_{1}+\ldots .+r_{n} a_{n}$. Similarly $(0, b) \notin \operatorname{acl}\left(\left(G^{\prime} \oplus\{0\}\right) \cup\right.$ $\left.\left\{\left(0, a_{1}\right), \ldots,\left(0, a_{n}\right)\right\}\right)$.

Since $\mathbb{G}_{S}^{\prime}$ is strongly minimal and $G^{\prime} \oplus\{0\} \prec \mathbb{G}_{S}^{\prime}$, there exists an automorphism $\tau$ of $\mathbb{G}_{S}^{\prime}$ which sends $(0, a)$ to $(0, b)$ and fixes pointwise $G^{\prime} \oplus\{0\}$ and $\left(0, a_{1}\right), \ldots,\left(0, a_{n}\right)$. From $\tau$ we construct easily an $L_{0}$-automorphism of $S$ which sends $a$ to $b$ and fixes $a_{1}, \ldots, a_{n}$ : just let $\sigma(s):=s^{\prime}$ if $\tau(0, s)=\left(x, s^{\prime}\right)$.

Let $a_{1}, \ldots, a_{n}$ and $b_{1}, \ldots, b_{n}$ be two tuples of $S$ which have the same $K$-vector space type. Assume that $a_{1}, . ., a_{k}$ are $K$-linearly independent and for every $j>k, a_{j}$ is dependent on $a_{1}, . ., a_{k}$. By the previous claim, there is an $L_{0}$-automorphism $\sigma$ of $S$ which sends $a_{1} a_{2} \ldots a_{k}$ on $b_{1} \ldots b_{k}$. But, then for every $j>k, \sigma\left(a_{j}\right)=b_{j}$.

Remark. If $\mathbb{G}$ is such that $G_{0}=\operatorname{acl}(\emptyset)=0$, then $\mathbb{T}=\mathbb{T}_{1}$ and $\mathbb{G}$ itself has the structure of a $K$-vector space. In that case, it will follows directly that $\mathbb{T}$ is finitely axiomatizable if and only if the theory of infinite $K$-vector spaces is finitely axiomatizable, and hence if and only if (see section 3.2.2) $K$ is finitely presented as a ring. 


\section{Application to finite axiomatizability}

In [17] Paljutin shows that "There exists a finitely axiomatizable, not locally finite categorical quasi-variety if and only if one of the following conditions hold:

1) there exists an infinite finitely presented ring which is a division ring;

2) there exists an infinite finitely presented group with a finite number of elements $g_{1}, \ldots, g_{n}$ such that every non trivial cyclic subgroup of $G$ intersects one of the conjugacy classes of the elements $g_{1}, \ldots, g_{n} . "$

The proof proceeds by showing first that, if there is a such a finitely axiomatizable quasi-variety (a quasi-variety is the class of models of a set of universal Horn sentences), then there exists one which is "standard", where the standard quasi-varieties are either $K$-vector spaces for a division ring $K$, or the Cayley graph of a group. Then he shows that if the quasi-variety of $K$-vector spaces is finitely axiomatizable and not $\omega$-categorical, $K$ must satisfy 1 ), and that if the Cayley graph of $G$ is finitely axiomatizable and not $\omega$-categorical, then $G$ must satisfy 2) (a proof of this part, due to M.A. Taitslin and Yu.E. Shimarev had already appeared in [1]).

The existence of such a ring and such a group are both still open. The existence of an infinite finitely presented group with finitely many conjugation classes is a well-known long standing open question, but the existence of the a priori weaker condition required in 2) is also open.

Concerning the existence of a ring satisfying 1), it seems that it is already unknown whether there exists an infinite finitely generated ring which is a division ring. One can only easily see that such a division ring cannot be commutative (see section 3.2.2 below).

For some years now A. Ivanov has been working on the conjecture that the existence of any finitely axiomatizable strongly minimal trivial set must imply the existence of a group satisfying 2$)$ and has proved some partial results $([12,13])$. The idea behind this conjecture is that such a group should appear as a subgroup or a quotient of the automorphism group of a connected component of the trivial strongly minimal set.

As explained in the introduction, Hrushovski then showed in [10] that any finitely axiomatizable $\aleph_{1}$-categorical theory must be locally modular and suggested the conjecture that if the theory has non trivial pregeometry, then the associated division ring of quasi-endomorphisms must satisfy 1).

In order to be quite self-contained on the subject of finite axiomatizability, and because sometimes a certain confusion arises on what exactly is meant by finite axiomatizability (in the case of an infinite language, for example), we will present in the next section precise definitions and basic transfer properties. For the same reasons, in section 3.2 , we will present a detailed exposition of the two "standard" cases.

\subsection{Transferring finite axiomatizability}

We are going to need to transfer the property of being finitely axiomatizable through various changes of languages and interpretations, and to be quite precise when we do it. We have unfortunately not found a completely adequate reference for our purpose, which 
we could have simply quoted or referred to. Most of what follows appears in various places under slightly different forms. The closest references for bi-interpretability can be found in [2] or, more recently, in [19]. In both cases the notions were used in the context of quasi-finitely axiomatizable $\omega$-categorical theories.

First, we need to recall precisely what it means for a theory in an infinite language to be finitely axiomatizable.

¿From now on, when we use the word theory, we mean a consistant set of axioms which is closed under deduction.

Definition 3.1. Let $L$ be an infinite language and $T$ a theory in $L$. We say that $T$ is finitely axiomatizable if there is a finite sub-language $L_{0}$ of $L$ such that any model $M$ of $T$ is interdefinable (Definition 2.3) with its reduct to $L_{0}$ and the theory $T_{L_{0}}$, the restriction of $T$ to the language $L_{0}$, is finitely axiomatizable in $L_{0}$. We then say that $T$ is finitely axiomatizable in the finite language $L_{0}$.

The following easy lemma will be useful later:

Lemma 3.2. Let $T$ be a theory in a language $L$, let $L_{0}$ be a sub-language of $L$ and let $\Sigma$ be a subset of sentences from $T$ with the following properties:

- for every predicate symbol $R(\bar{x})$ in the language $L, \Sigma$ includes a sentence of the form $\forall \bar{x}\left(\phi_{R}(\bar{x}) \leftrightarrow R(\bar{x})\right)$ where $\phi_{R}(x)$ is a formula in $L_{0}$,

- for every function symbol $f(\bar{x})$ in $L, \Sigma$ includes a sentence of the form $\forall \bar{x} \forall y\left(\phi_{f}(\bar{x}, y) \leftrightarrow\right.$ $f(\bar{x})=y$ ) where $\phi_{f}(\bar{x}, y)$ is a formula in $L_{0}$,

- for every constant symbol $c$ in $L, \Sigma$ includes a sentence of the form $\forall x\left(\phi_{c}(x) \leftrightarrow\right.$ $x=c)$ where $\phi_{c}(x)$ is a formula in $L_{0}$.

Then $T$ is axiomatized by $\Sigma \cup T_{L_{0}}$.

Finite axiomatizability is easily seen to transfer through interdefinability.

Lemma 3.3. Let $\mathcal{M}_{1}=\left(M, L_{1}\right)$ and $\mathcal{M}_{2}=\left(M, L_{2}\right)$ be two structures on the same domain $M$ which are interdefinable. Then $T h\left(\mathcal{M}_{1}\right)$ is finitely axiomatizable if and only if $T h\left(\mathcal{M}_{2}\right)$ is finitely axiomatizable.

First some basic remarks about extending the language while keeping finite axiomatizability:

Lemma 3.4. (i) Let $\mathcal{M}=(M, L)$ be a structure in a finite language $L$. If $E$ is a $\emptyset$-definable equivalence relation in $\mathcal{M}$ then the theory of $\mathcal{M}$ has a finite axiomatization if and only if the theory of the reduct of $\mathcal{M}^{e q}, \mathcal{M}_{E}=\left(M, M^{n} / E, L, f_{E}\right)$ has a finite axiomatization.

(ii) Let $\mathcal{M}=(M, L)$ be a structure in a finite language L. Let $\bar{a}$ be a tuple of $M$ such that the type of $\bar{a}$ is isolated. Then the theory of $\mathcal{M}$ has a finite axiomatization if and only if the theory of $\mathcal{M}_{\bar{a}}=(M, L, \bar{a})$ has a finite axiomatization.

We now check that one can reduce questions of finite axiomatizability for $\aleph_{1}$-categorical one-based groups to the case of abelian structures.

Recall the fundamental properties of one-based groups (the reader can take Property 1 as a definition for one-based groups) 
Fact 3.5. [11] 1. A group $\mathcal{G}$ is one-based if and only if, for every $n \geq 1$, every definable subset of $G^{n}$ is a Boolean combination of definable cosets of connected definable subgroups of $G^{n}$.

2. A one-based group is definably abelian by finite, that is, has a definable normal abelian subgroup of finite index.

3. Let $\mathcal{H}$ be a one-based group. Let $S \subset H^{n}$ be a definable connected subgroup. Then $S$ is definable over acl ${ }^{e q}(\emptyset)$.

Corollary 3.6. Let $\mathcal{G}$ be an $\aleph_{1}$-saturated one-based group. Fix $\mathcal{M}_{0}$ a countable elementary sub-model of $\mathcal{G}$. Let $A \subset G$; any $A$-definable subset of $G^{n}$ is a Boolean combination of $A$-definable cosets of some $M_{0}$-definable connected subgroups.

Now let $\mathcal{G}=(G, L)$ be an $\aleph_{1}$-saturated connected one-based group. Fix some countable elementary sub-model of $\mathcal{G}, M_{0}$. Let $\left(H_{i}\right)_{i \in I}$ be the family of all connected $M_{0^{-}}$ definable subgroups in $\bigcup_{n \geq 1} G^{n}$. Let $\mathbb{G}$ be the following abelian structure:

$$
\mathbb{G}=<G,+,-, 0,\left(H_{i}\right)_{i \in I},(m)_{m \in M_{0}}>\text {. }
$$

Then it follows from Fact 3.5 and Corollary 3.6 that $\mathcal{G}$ and $\mathbb{G}$ are $M_{0}$-interdefinable.

Corollary 3.7. Let $\mathcal{G}$ be an $\omega$-stable one-based connected group such that $T h(\mathcal{G})$ is finitely axiomatizable in a finite language $L$. Then there is a finitely axiomatizable abelian structure with constants $\mathbb{G}$, which is interdefinable with a finitely axiomatizable expansion of $\mathcal{G}$ by finitely many constants.

Proof. Without loss of generality, suppose that $\mathcal{G}$ is $\aleph_{1}$-saturated. Consider $\mathcal{M}_{0} \preceq \mathcal{G}$, the prime model of $T h(\mathcal{G})$, by $\omega$-stability. Consider the abelian structure $\mathbb{G}$ described above which is $M_{0}$-interdefinable with $\mathcal{G}$. As the language $L$ is finite, we can choose a finite family $H_{1}, \ldots, H_{k}$ and a finite sequence $m_{0}, \ldots, m_{n}$ of elements from the prime model $M_{0}$, such that every symbol from the language $L$ can be defined in the restriction $\mathbb{G}_{1}$ of $\mathbb{G}$ to the finite language

$$
L_{1}:=\left\{+,-, 0,\left(H_{i}\right)_{1 \leq i \leq k},\left\{m_{0}, \ldots, m_{n}\right\}\right\}
$$

and such that every $H_{i}$ is definable in $\mathcal{G}$ over $\left\{m_{0}, \ldots, m_{n}\right\}$. Now add $\left\{m_{0}, \ldots, m_{n}\right\}$ as new constants to the language $L$ of $\mathcal{G}$. As $M_{0}$ is atomic, the tuple $\left(m_{0}, \ldots, m_{n}\right)$ has isolated type over $\emptyset$ in $L$. By Lemma 3.4, the theory of the expansion $\mathcal{G}^{\prime}$ of $\mathcal{G}$ to $L^{\prime}:=L \cup\left\{m_{0}, \ldots, m_{n}\right\}$ remains finitely axiomatizable. Now the structures $\mathcal{G}^{\prime}$ and $\mathbb{G}_{1}$ are interdefinable (over $\emptyset$ ). Hence by Lemma $3.3 T h\left(\mathbb{G}_{1}\right)$ is finitely axiomatizable.

We will also need to check that an $\aleph_{1}$-categorical group is finitely axiomatizable if and only if its connected component is. This is a particular case of the transfer of finite axiomatizability by bi-interpretability.

Recall the definition of bi-interpretability from [2], or [19]:

Definition 3.8. Let $\mathcal{M}=\left(M, L_{1}\right)$ and $\mathcal{N}=\left(N, L_{2}\right)$. We say that $\mathcal{M}$ is $\emptyset$-interpretable in $\mathcal{N}$ if there is a $\emptyset$-definable subset $U$ of $N^{n}$, and a surjective map $f$, from $U$ onto $M$ such that: 
- the equivalence relation $E_{f}$ on $U \times U$ defined, for $(a, b) \in U \times U$, by $f(a)=f(b)$ is $\emptyset$-definable in $\mathcal{N}$,

- for every $k$-ary relation symbol $R$ in $L_{1}$, the subset $R_{f}$ of $U^{k}, R_{f}:=\left\{\left(a_{1}, \ldots, a_{k}\right) \in\right.$ $\left.U^{k} ; \mathcal{M} \models R\left(f\left(a_{1}\right), \ldots, f\left(a_{k}\right)\right)\right\}$ is $\emptyset$-definable in $\mathcal{N}$

- for every $k$-ary function symbol $h$ in $L_{1}$, the subset $G r_{f}(h):=\left\{\left(a_{1}, \ldots, a_{k}, b\right) \in\right.$ $\left.U^{k+1} ; \mathcal{M} \models g\left(f\left(a_{1}\right), \ldots, f\left(a_{k}\right)\right)=f(b)\right\}$ is $\emptyset$-definable in $\mathcal{N}$,

- for every constant symbol $c$ in $L_{1}$, the subset $c_{f}:=\{a \in U: \mathcal{M} \models f(a)=c\}$ is $\emptyset$-definable in $\mathcal{N}$.

It follows that $f$ induces an isomorphism of $L_{1}$-structures between $U / E_{f}$ (subset of $N^{e q}$ ) and $\mathcal{M}$.

If $\mathcal{M}$ is interpretable in $\mathcal{N}$, via the surjective map $f$ from $U \subset N^{k}$ onto $M$, and $\mathcal{N}$ is interpretable in $\mathcal{Q}=\left(Q, L_{3}\right)$, via the surjective map $g$ from $V \subset Q^{n}$ onto $N$, let $W:=\left\{\left(q_{1}, \ldots, q_{k}\right) \in\left(Q^{n}\right)^{k}:\left(g\left(q_{1}\right), \ldots, g\left(q_{k}\right)\right) \in U\right\}$. We denote by $f \circ g$ the obvious induced map from $W \subset S^{n k}$ onto $M$. One checks easily that $f \circ g$ is an interpretation of $\mathcal{M}$ in $\mathcal{Q}$.

If $\mathcal{M}$ is interpretable in $\mathcal{N}$, via $f$, and $\mathcal{N}$ is interpretable in $\mathcal{M}$ via $g$, we say that $\mathcal{M}$ and $\mathcal{N}$ are bi-interpretable if $f \circ g$ is a $\emptyset$-definable map in $\mathcal{M}$ and $g \circ f$ a $\emptyset$-definable map in $\mathcal{N}$.

We leave the checking of the following lemma to the reader.

Lemma 3.9. Let $\mathcal{M}$ be an $L_{1}$-structure and $\mathcal{N}$ an $L_{2}$-structure which are bi-interpretable. Then $T h(\mathcal{M})$ is finitely axiomatizable if and only if $T h(\mathcal{N})$ is.

We recall the definition of an induced structure on a definable subset:

Definition 3.10. Let $\mathcal{M}$ be an $L$-structure and $D$ be a $\emptyset$-definable subset of $M^{n}$. The

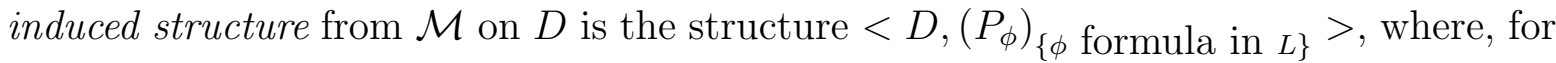
$\phi\left(x_{1}, \ldots, x_{k}\right),\left|x_{j}\right|=n, P_{\phi}$ is a predicate of arity $k$ which is interpreted on $D$ by the set $D^{k} \cap \phi\left(M^{n k}\right)$.

Lemma 3.11. Let $\mathcal{M}$ be an L-structure which is the union of a finite definable partition, that is, $M=M_{1} \cup \ldots \cup M_{n}$, where, for $1 \leq i<j \leq n, M_{i}$ is $\emptyset$-definable and $M_{i} \cap M_{j}=\emptyset$. Suppose furthermore that for each $i>1$ there is a $\emptyset$-definable bijection, $f_{i}$ from $M_{1}$ onto $M_{i}$ and that $L$ contains $n$ constant symbols $\left\{c_{1}, \ldots, c_{n}\right\}$ which are interpreted in $\mathcal{M}$ by distinct elements of $M_{1}$. Consider $M_{1}$ together with its induced structure from $\mathcal{M}$, denoted $\mathcal{M}_{1}$. Then $\mathcal{M}$ and $\mathcal{M}_{1}$ are bi-interpretable.

Proof. Let $U:=M_{1} \times\left\{c_{1}, \ldots, c_{n}\right\} \subset M_{1}^{2}$ and let $f: U \mapsto M, f\left(x, c_{i}\right)=f_{i}(x)$, where $f_{1}$ is the identity on $M_{1}$. Then $f$ gives an (injective) interpretation of $\mathcal{M}$ into $\mathcal{M}_{1}$. Indeed, for any $k$-ary predicate symbol $R$ from $L$, the set $R_{f}=\left\{\left(a_{1}, \ldots, a_{k}\right) \in U: \mathcal{M} \models\right.$ $R\left(f\left(a_{1}\right), \ldots, f\left(a_{k}\right)\right\}$ is a basic predicate in the language of the induced structure, hence certainly definable. Similarly for the other symbols of $L$.

Let $g: M_{1} \subset M \mapsto M_{1}$ be the identity. Let $W:=\left\{(x, y) \in U: f(x, y) \in M_{1}\right\}=$ $M_{1} \times\left\{c_{1}\right\}$. Then $g \circ f: W \mapsto M_{1}$ is an interpretation of $\mathcal{M}_{1}$ into itself. For $\left(x, c_{1}\right) \in U$, $g \circ f\left(x, c_{1}\right)=x$ is the first projection and is hence $\emptyset$-definable in $\mathcal{M}_{1}$. 
Let $W^{\prime}:=\left\{(x, y) \in M_{1} \times M_{1}:(g(x), g(y)) \in U\right\}$. Then $W^{\prime}=U=M_{1} \times\left\{c_{1}, \ldots, c_{n}\right\}$, and $f \circ g\left(x, c_{i}\right)=f_{i}(x)$ is $\emptyset$-definable in $\mathcal{M}$.

Corollary 3.12. Let $\mathcal{G}=(G, L)$ be an $\omega$-stable group in a language $L$. Let $\mathcal{G}^{0}$ be the connected component of $G, G^{0}$, with the induced structure from $\mathcal{G}$. Then $T h(\mathcal{G})$ is finitely axiomatizable if and only if $T h\left(\mathcal{G}^{0}\right)$ is finitely axiomatizable.

Proof. By $\omega$-stability, $G^{0}$ is $\emptyset$-definable in $\mathcal{G}$ and has finite index in $G$. By $\omega$-stability again, there is a prime model $G_{1}, G_{1} \preceq \mathcal{G}$, atomic over $\emptyset$. Choose $a_{1}, \ldots, a_{n}$ in this prime model such that $G=a_{1} G^{0} \cup \ldots \cup a_{n} G^{0}$, with $a_{1}=1$. Choose $c_{1}, \ldots c_{n}$ distinct elements from $G_{1}{ }^{0}$, the connected component of $G_{1}$. Then the type of the tuple $a_{1}, \ldots, a_{n}, c_{1}, \ldots, c_{n}$ is isolated over the empty set. Let $T$ denote the complete theory of $\mathcal{G}$ in the language $L$ together with $\left\{a_{1}, \ldots, a_{n}, c_{1}, \ldots, c_{n}\right\}$ added as new constants. By 3.4, $T h(\mathcal{G})$ (in $L$ ) is finitely axiomatizable if and only if $T$ is. Similarly, let $T_{0}$ be $T h\left(\mathcal{G}^{0}\right)$ in the language with $\left\{c_{1}, \ldots, c_{n}\right\}$ added as new constants, then $T h\left(\mathcal{G}^{0}\right)$ is finitely axiomatizable iff $T_{0}$ is. We are now in the situation of Lemma 3.11: $G$ is the union of a finite $\emptyset$-definable partition $a_{1} G^{0} \cup \ldots \cup a_{n} G^{0}$, and for each $i$, there is a $\emptyset$-definable bijection $f_{i}$ from $a_{i} G^{0}$ onto $a_{1} G^{0}=G^{0}, f_{i}\left(a_{i} g\right)=g$. The result then follows by 3.11 and 3.9 .

\subsection{The classical examples}

Before we start on the description of the two emblematic examples, we would like to draw the reader's attention to the following: if $T$ is a theory in $L$ which is finitely axiomatizable in a finite sub-language $L_{0}$ of $L$ then it is certainly finitely axiomatizable in every finite sub-language $L_{1}$ of $L$ containing $L_{0}$ (Lemma 3.2), but one should be a little careful. For example suppose that $T$ is a complete theory in an infinite language $L$, which is finitely axiomatizable in a finite sub-language $L_{0}$ of $L$. Let $\mathbb{F}_{0}$ be a finite set of axioms for $T_{L_{0}}$, and let $\Sigma$ be an arbitrary infinite set of axioms in $L$ for the complete theory $T$. By compactness some finite subset $\Sigma_{1}$ of $\Sigma$ will axiomatize $T_{L_{0}}$. But if $L_{1}$ is the finite sub-language of $L$ containing all symbols appearing in $\Sigma_{1}$, there is no reason that $\Sigma_{1} \vdash T_{L_{1}}$, or equivalently there is no reason for $\Sigma_{1}$ to axiomatize a complete theory in the language $L_{1}$. This explains the care taken in identifying the right set of axioms in the following proofs.

\subsubsection{The trivial example}

First, recall that for any non trivial group $G$, the theory $T_{G}$ which describes $G$ acting semi-regularly (the stabilizer of every element is trivial) on an infinite set, in the language $L_{G}:=\{g: g \in G\}$, where each $g$ is a unary function symbol, is strongly minimal, eliminates quantifiers and has trivial geometry. The theory $T_{G}$ is $\omega$-categorical if and only if $G$ is finite. If $G$ is infinite, the Cayley graph of $G$ (that is the regular action of $G$ on itself by left multiplication) is a model of $T_{G}$. The theory $T_{G}$ can be axiomatized by the following set of axioms, $\Sigma_{G}$, if $G$ is infinite:

$$
-\forall x 1(x)=x ;
$$


- $\forall x g(x) \neq x$, for every $g \neq 1 \in G$;

- $\forall x g(h(x))=r(x)$, for every $g, h, r \in G$ such that $g h=r$.

If $G$ is finite, then $T_{G}$ can be axiomatized by $\Sigma_{G}$ together with the scheme for infinity.

Note that, for any model $M$ of $T_{G}$, for any $a \in M$, the definable closure of $\{a\}$ in $M$ in the language $L_{G}$ is the $G$-orbit of $a$.

Now suppose that we have a presentation of $G$, i.e. that $G$ is isomorphic to the free group on $S=\left\{s_{i} ; i \in I\right\}$ modded out by a normal subgroup $P$. Then the theory $T_{G}$ is clearly interdefinable with the following theory in the language $L_{S}=\{s: s \in S\}$, which we denote by $\Sigma_{S}$ : let $W$ be the set of words on $\mathrm{S}$,

$-\forall x w(x)=x$ for every $w \in P$,

$-\forall x w(x) \neq x$ for every $w \in W \backslash P$.

Suppose that $G$ is an infinite finitely presented group $G$, with a finite number of conjugation classes $C_{1}, \ldots C_{k}$ such that every non trivial cyclic subgroup of $G$ intersects one of the $C_{i}$ 's. Then $T_{G}$ is finitely axiomatizable (in the sense of Definition 3.1). Indeed, choose $F=\left\{g_{1}, \ldots, g_{n}\right\} \subset G$ such that: for every $j, g_{j} \neq 1, F$ generates $G$, there is a finite set $P_{0}$ of words on $F$ which generates the presentation of $G, P, F$ is closed under inverse, and for every $g \in G \backslash\{1\}$, there is some $m>0$ such that $g^{m}$ is conjugate to one of the $g_{j}$ 's. Let $\Sigma_{F}$ be the (complete) set of axioms described above, in the finite language $L_{F}:=\left\{g_{1}, \ldots, g_{n}\right\}$, which is interdefinable with $T_{G}$. Consider $\Sigma_{0}$, the following finite subset of $\Sigma_{F}$ :

$-\forall x g_{j}(x) \neq x$, for every $j, 1 \leq j \leq n$,

$-\forall x w(x)=x$, for every $w \in P_{0}$.

We must check that $\Sigma_{0}$ is an axiomatization for $\Sigma_{F}$. If $w \in P$, then, for all $x, w(x)=1$ as $P$ is the normal subgroup generated by $P_{0}$. If $g$ is any word on $F$, and $g \notin P$, we must check that for all $x, g(x) \neq x$. By assumption, there are $m>0, g_{j} \in F$ and $h \in G$, such that $g^{m}=h^{-1} g_{j} h$. If $g(x)=x$ for some $x$, then $g^{m}(x)=x=h^{-1}\left(g_{j}(h(x))\right.$, hence $h(x)=g_{j}(h(x))$. But this contradicts $\Sigma_{0}$.

Conversely, suppose that $G$ is infinite and that the theory $T_{G}$ is finitely axiomatizable. Let $F \subset G$, be finite such that $T:=T_{G}$ is finitely axiomatizable in the sub-language $L_{F}:=\{f ; f \in F\}$, that is, such that any model of $T_{G}$ is interdefinable with its reduct to $L_{F}$, and the (complete) theory $T_{G} \mid L_{F}$ is finitely axiomatizable. Let $H$ be the subgroup of $G$ generated by $F$. Then $T_{G} \mid L_{H}$ contains the theory $T_{H}$, which is complete, hence it is equal to $T_{H}$. In the language $L_{G}, G$ which is a model of $T_{G}$, is equal to the definable closure of the identity element 1 . Similarly, $H$ is, in $L_{H}$ the definable closure of 1 . By interdefinablity of $L_{G}$ with $L_{F}, G$ is also equal to the definable closure of 1 in $L_{F}$, hence also in $L_{H}$. It follows that $G=H$.

So we know that $G$ is finitely generated, hence isomorphic to the free group on a finite set of generators $S$, which we suppose closed under inverse, modded out by a normal subgroup $P$. Let $W$ be the set of all words on $F$. Pass to the theory $T_{S}$ (axiomatized by $\Sigma_{S}$ ) in the finite language $\{s: s \in S\}$, which is interdefinable with $T_{G}$, By finite axiomatizability, there is a finite subset $W_{0}$ of $W$ (the set of words on $S$ ) such that $\Sigma_{S}$ can be axiomatized by $\Sigma_{W_{0}}$ : 
$-\forall x w(x)=x$ for every $w \in W_{0} \cap P$,

$-\forall x w(x) \neq x$ for every $w \in W_{0} \backslash P$.

We can suppose that, for every $s \in S, s s^{-1} \in W_{0}$.

Let $N$ be the normal subgroup generated by $W_{0} \cap P$ in $D$, the free group on $S$. By construction $N \subset P$. The Cayley graph of $D / N$, in the language $L_{S}$ is a model of $\Sigma_{W_{0}}$, hence is interdefinable with a model of $\Sigma_{S}$. It follows that $N=P$ and $G \cong D / N$.

Let $h \in G, h \neq 1$, let $H$ denote the subgroup generated by $h$ in $G$, and $G / H$ the set of left cosets, equipped with an $L_{G}$ structure by the left action of $G$. As the action of $G$ is not semi-regular on $G / H, G / H$ is not a model of $\Sigma_{S}$, hence by finite axiomatizability, it is not a model of $\Sigma_{W_{0}}$. So there is some $g \in W_{0} \backslash N$ and some coset $a H$ such that $g(a H)=a H$, that is, such that $a^{-1} g a=h^{n}$ for some integer $n$. So any non trivial element $h$ has a power which is conjugate to one of the $g^{\prime} s$ in $W_{0}$.

\subsubsection{Vector spaces}

Let $K$ be any countable division ring. Let $L_{K}$ be the usual language for $K$-vector spaces, $L_{K}:=\left\{+,-, 0,(k)_{k \in K}\right\}$, where $k$ is a unary function interpreted as scalar multiplication by the element $k$. Consider $T_{K}$ the theory of all infinite $K$-vector spaces in $L_{K}$. The theory $T_{K}$ is $\aleph_{1}$-categorical and is totally categorical if and only if $K$ is finite.

Suppose that $K$ is an infinite division ring which is finitely presented as a ring. Then the complete theory of $K$-vector spaces is finitely axiomatizable in the following way: let $F$ be a finite subset of $K$, which generates $K$ as a ring and such that there is a finite set of terms in $F, P$, which generates the presentation of $K$ (a two-sided ideal $J$, such that $K$ is isomorphic to the quotient fo the free ring generated by $F$ BY $J$ ). Then $T_{K}$ is finitely axiomatized in $L_{F}:=\left\{+,-, 0,1,(f)_{f \in F}\right\}$ by

- axioms for abelian groups

$-\forall x 1(x)=x$,

$-\forall x \forall y f(x+y)=f(x)+f(y)$, for every $f \in F$

- $\forall x w(x)=0$, for every $w \in P$.

For the converse, we now suppose that the theory of infinite $K$-vector spaces is finitely axiomatizable. By the classical results on the non finite axiomatizability of totally categorical theories, the assumption forces $K$ to be infinite.

Proposition 3.13. Let $K$ be an infinite division ring. If the theory of $K$-vector spaces is finitely axiomatizable then $K$ is finitely presented as a ring.

Proof. Let $T_{K}$ be the theory of non trivial $K$-vector spaces, in the usual language $L_{K}=$ $\{0,+,-, k: k \in K\}$.

Let $X$ be a finite subset of $K$ such that $T_{K}$ is finitely axiomatizable in the finite language $L_{X}:=\{0,+,-, k: k \in X\}$.

Claim. $K$ is generated as a skew field by $X$. 
Proof. Let $K_{0}$ be the subfield of $K$ generated by $X$. Then the theory $T_{K_{0}}$ of infinite $K_{0^{-}}$ vector spaces is a subset of $T_{\mid L_{K_{0}}}$ and since $T_{K_{0}}$ is complete, they are equal. Now, consider $K$ as a $K$-vector space. Then $K=\operatorname{dcl}_{L_{K}}\left(1_{K}\right)=\operatorname{dcl}_{L_{X}}\left(1_{K}\right)=\operatorname{dcl}_{L_{K_{0}}}\left(1_{K}\right)=K_{0}$.

Denote by $S_{K}$ the classical axiomatization of $T_{K}$ :

1. $\exists x x \neq 0$;

2. axioms for abelian groups;

3. $\forall x 1_{K}(x)=x$;

4. $\forall x \forall y k(x+y)=k(x)+k(y),(k \in K)$;

5. $\forall x k(x)+k^{\prime}(x)=k^{\prime \prime}(x),\left(k, k^{\prime}, k^{\prime \prime} \in K, k^{\prime \prime}=k+k^{\prime}\right)$;

6. $\forall x k\left(k^{\prime}(x)\right)=k^{\prime \prime}(x),\left(k, k^{\prime}, k^{\prime \prime} \in K, k^{\prime \prime}=k k^{\prime}\right)$.

If $A$ is a subset of $K$ we will denote by $S_{A}$ the subset of sentences of $S_{K}$ in the language $L_{A}:=\{0,+,-, k: k \in A\}$. A priori, $S_{X}$ does not give an axiomatization of the complete theory $T_{L_{X}}$, but since there is some finite axiomatization of $T_{L_{X}}$ by assumption, there exists by compactness a finite subset $Y$ containing $X$ such that $S_{Y}$ implies $T_{L_{X}}$.

We are going to enlarge $Y$ in order that $S_{Y}$ implies the complete theory $T_{L_{Y}}$. First we define the depth of elements of $K$ relatively to $X$. We assume that $1_{K}, O_{K} \in X$ and we define by induction a sequence $\left(W_{i}\right)_{i \in \omega}$ of subsets of $K$ such that $W_{0}:=X$ and

$$
W_{i+1}:=\left\{k \in K: k=-k_{1} \text { or } k=k_{1}^{-1} \text { or } k=k_{1}+k_{2} \text { or } k=k_{1} k_{2} \text { for } k_{1}, k_{2} \in W_{i}\right\} .
$$

Then $K=\cup_{i \in \omega} W_{i}$ since $X$ generates $K$ as a skew field $K$. We define the depth of $k \in K$ as the smallest integer $n$ such that $k \in W_{n}$. Now by an easy induction, we can enlarge $Y$ so that it remains finite and for each $k \in Y$, if the depth of $k$ is $n+1$ then there exists $k_{1}, k_{2} \in Y$ of depths at most $n$ such that $k=-k_{1}$, or $k=k_{1}^{-1}$, or $k=k_{1}+k_{2}$ or $k=k_{1} k_{2}$.

Claim. Then $T_{L_{Y}}$ is axiomatized by $S_{Y}$.

Proof. We choose, by induction on the depth of elements of $Y$, for each $k \in Y$, a formula $\phi_{k}(x, y) \in L_{X}$ such that $S_{Y} \vdash \forall x \forall y(k x=y) \leftrightarrow \phi_{k}(x, y)$ (we know by assumption that there is such a formula for which $T \vdash \forall x \forall y(k x=y) \leftrightarrow \phi_{k}(x, y)$, but we want one such that the equivalence can be deduced from $S_{Y}$ ). If $k \in Y$ has depth 0 (i.e. $k \in X$ ), then we let $\phi_{k}(x, y):=(k x=y)$. Assume that we have chosen a formula $\phi_{k}$ for each $k \in Y$ of depth less or equal to $n$. Let $k \in Y$ have depth $n+1$. Then there exist $k_{1}, k_{2} \in Y$, of depth at most $n$, such that at least one of the following cases occur:

- $k=-k_{1}$; in this case we let $\phi_{k}(x, y):=\phi_{k_{1}}(x,-y)$,

- or $k=k_{1}^{-1}$; in this case we let $\phi_{k}(x, y):=\phi_{k_{1}}(y, x)$, 
- or $k=k_{1}+k_{2}$; in this case we let

$$
\phi_{k}(x, y):=\exists t_{1} \exists t_{2}\left(\phi_{k_{1}}\left(x, t_{1}\right) \wedge \phi_{k_{2}}\left(x, t_{2}\right) \wedge\left(y=t_{1}+t_{2}\right)\right),
$$

- or $k=k_{1} k_{2}$; in this case we let

$$
\phi_{k}(x, y):=\exists t\left(\phi_{k_{2}}(x, t) \wedge \phi_{k_{1}}(t, y)\right) .
$$

Since $T_{K}$ is finitely axiomatizable in the language $L_{X}$ and for each $k \in Y, T_{L_{Y}} \vdash$ $\forall x \forall y(k x=y) \leftrightarrow \phi_{k}(x, y)$, the complete theory $T_{L_{Y}}$ is axiomatized by

$$
T_{L_{X}} \cup\left\{\forall x \forall y(k x=y) \leftrightarrow \phi_{k}(x, y): k \in Y\right\} .
$$

It follows that $S_{Y}$ is an axiomatization of $T_{L_{Y}}$.

Now, we are going to prove that $K$ is isomorphic to the finitely presented $\operatorname{ring} A$, given by the set of generators $\{k: k \in Y\}$ and the presentation:

$$
\left\{1_{K}-1\right\} \cup\left\{k_{1}+k_{2}-k_{3}: k_{1}, k_{2}, k_{3} \in Y ; k_{3}=k_{1}+k_{2}\right\} \cup\left\{k_{1} k_{2}-k_{3}: k_{1}, k_{2}, k_{3} \in Y ; k_{3}=k_{1} k_{2}\right\} .
$$

Remark that every non trivial $A$-module is a model of $S_{Y}$ as an $L_{Y}$-structure, hence any two non trivial $A$-modules are elementarily equivalent in the language $L_{Y}$. Furthermore, any non trivial $A$-module has a canonical expansion to a $K$-vector space : by assumption, for each $k \in K$, there is a formula $\theta_{k} \in L_{X} \subset L_{Y}$, such that $T \vdash \forall x \forall y(k x=y) \leftrightarrow$ $\theta_{k}(x, y)$. Define, for $k \in K, m, n \in M, k m=n$ iff $M \models \theta_{k}(m, n)$.

Let $\psi$ be the canonical morphism from $A$ to $K$ which sends each generator $k \in Y$ to $k \in K$. The morphism $\psi$ is injective: consider the $A$-module structure on $K$ given via $\psi$, i.e. define $a x:=\psi(a) x$. As $A$-modules, $K$ and $A$ are elementarily equivalent. In $A$, if $a \neq 0$, then for some $x, a x \neq 0$, hence this is also true in $K$, which implies that $\psi(a) \neq 0$.

Hence $A$ has no zero divisors. Again by completeness of the theory of non trivial $A$-modules, this implies that in all non trivial $A$-modules, if $a \neq 0 \in A$, if $x \neq 0$, then $a x \neq 0$. This implies that $A$ is a division ring: if $a \in A \backslash\{0\}$ was not left invertible, $A / A a$ would be a non-trivial $A$-module satisfying that $a x=0$ for some $x \neq 0(x=1+A a)$. Since $X \subset \psi(A), X$ generates $K$ as a skew field and $\psi(A)$ is a skew field, we obtain that $\psi(A)=K$.

Remark. As we have mentioned above, it seems to be an open question whether there exists an infinite division ring which is finitely generated as a ring. It is easily seen, though, that there is no such commutative division ring: Let $K$ be a field which is finitely generated as a ring, and let $k$ denote its prime field $\left(k=\mathbb{F}_{p}\right.$ or $\left.k=\mathbb{Q}\right)$. As $K$ is finitely generated as a ring over $k$, then $K$ is contained in $k^{a l g}$, the algebraic closure of $k$. If $K$ has characteristic $p>0$, then $K=\mathbb{F}_{p}\left[a_{1}, \ldots, a_{n}\right]$ is finite. Otherwise $k=\mathbb{Q}$, and there are $a_{1}, \ldots, a_{n} \in \mathbb{Q}^{\text {alg }}$, such that $K=\mathbb{Z}\left[a_{1}, \ldots, a_{n}\right]$. In that case, for some integer $m>0$, the $a_{i}$ 's are entire over $A:=\mathbb{Z}[1 / m]$ and $K$ is finitely generated as an $A$-module. As $A$ is Noetherian, $K$ is Noetherian as an $A$-module, and $\mathbb{Q}$, as an $A$-submodule, must also be finitely generated, which is impossible. 


\subsection{Finitely axiomatizable strongly minimal abelian structures}

We suppose that $\mathbb{G}$ is a strongly minimal abelian structure in a finite language $L_{c}=$ $L_{0} \cup\{c \in C\}$ such that its theory $\mathbb{T}$ is finitely axiomatizable. Recall from corollary 1.4 that $\mathbb{T}$ is axiomatized by the set of sentences $T(\mathbb{G})$ together with the pp-type of the constants. Let $\mathbb{B}$ be a finite axiomatization of $\mathbb{T}$ which consists of a finite subset $\mathbb{A}$ of $T(\mathbb{G})$ together with a finite subset of the $p p$-type of the constants. Denote by $\mathbb{A}_{1}$ the following finite subset of $\mathbb{T}_{1}$ (as defined in section 1.8):

- the axioms for abelian groups,

- for each original predicate $H$ from $L_{0}, H$ is a subgroup,

- the equivalence sentences in $\mathbb{A}$,

- $[\phi: \psi] \geq k$ for every $k$ and for every pair of $p p$-formulas such that in $\mathbb{G}, \psi(G) \subset$ $\phi(G) \subset G,[\phi(G): \psi(G)]$ is infinite and the sentence $[\phi: \psi] \geq k$ is in $\mathbb{A}$,

- $[\phi: \psi]=1$ for every pair of $p p$-formulas such that in $\mathbb{G}, \psi(G) \subset \phi(G) \subset G$ and for some integer $k$, the sentence $[\phi(G): \psi(G)]=k$ is in $\mathbb{A}$,

- $\exists x \neq 0$.

Lemma 3.14. For every model $\mathbb{G}$ of $\mathbb{T}$ and every model $\mathbb{S}$ of $\mathbb{A}_{1}$, the $L_{c}$-structure $\mathbb{G}_{S}$ $(=\mathbb{G} \oplus \mathbb{S}$ as in section 1.2) is a model of $\mathbb{B}$ and so, of $\mathbb{T}$. Moreover, $G \oplus\{0\}$ is an elementary submodel of $\mathbb{G}_{S}$.

Proof. The proof that $\mathbb{G}_{S}$ is a model of $\mathbb{B}$ is exactly similar to the proof of 1.15 . One needs to check the dimensions only for the $p p$-subgroups $\phi, \psi$ such that a dimension sentence of the form $[\phi: \psi] \geq n$ (if it is infinite) or $[\phi: \psi]=n$ (if it is finite) appears in $\mathbb{A}$.

We are going to show that $\mathbb{A}_{1}$ gives an axiomatization for the complete theory $\mathbb{T}_{1}$. It suffices to show that every model of $\mathbb{A}_{1}$ is infinite:

Lemma 3.15. If all models of $\mathbb{A}_{1}$ are infinite then $\mathbb{A}_{1}$ is a finite axiomatization of $\mathbb{T}_{1}$.

Proof. We show that any two models of $\mathbb{A}_{1}$ of cardinality $\aleph_{1}$ are isomorphic, then if $\mathbb{A}_{1}$ has no finite models, it is complete and hence axiomatizes $\mathbb{T}_{1}$. Let $\mathbb{G}$ be a countable model of $\mathbb{T}$. (One can choose $\operatorname{acl}(\emptyset)$ if it is infinite.) Let $\mathbb{S}_{1}$ and $\mathbb{S}_{2}$ be two models of $\mathbb{A}_{1}$ of cardinality $\aleph_{1}$. Then by strong minimality, as $G \oplus\{0\}$ is algebraically closed in $\mathbb{G}_{S_{i}}$, there is an isomorphism between $\mathbb{G}_{S_{1}}$ and $\mathbb{G}_{S_{2}}$ which is the identity on $G \oplus\{0\}$. From this isomorphism one induces easily an isomorphism between $\mathbb{S}_{1}$ and $\mathbb{S}_{2}$.

Proposition 3.16. The ring of quasi-endomorphisms of $\mathbb{G}$ is infinite.

Proof. Suppose not. Let $K$ denote the ring of quasi-endomorphisms, then $K=\mathbb{F}_{q}$ and $G$ has bounded exponent. In particular every finitely generated subgroup of $G$ is finite. We are going to construct a finitely generated subgroup of $G$ which is a model of $\mathbb{T}$, contradicting the completeness of $\mathbb{T}$.

First, we add the quasi-endomorphisms as predicates to the language: for each $\alpha \in$ $\mathbb{F}_{q}$, denote by $H_{\alpha}$ the corresponding quasi-endomorphism, which is a strongly minimal 
subgroup of $G^{2}$, definable over $\emptyset$ by Lemma 1.6, such that its first projection is equal to $G$. We add to $L_{c}$ a predicate $\hat{H}_{\alpha}$ for each $\alpha \in \mathbb{F}_{q}$. This preserves the finite axiomatizability of $\mathbb{G}$. So we can assume that the language $L_{c}$ already contains the quasi-endomorphisms as predicates. Now, we also, if necessary, add finitely many new predicates for some $p p$-definable subgroups which appear in the axiomatization $\mathbb{B}$, so that $\mathbb{T}$ has a finite axiomatization containing only sentences of the following type where $X, Y$ and $Z$ are amongst the predicates $\hat{H}_{i}$ of $L_{c}$ :

1. $G$ is a group;

2. the $\hat{H}_{i}$ 's are subgroups;

3. the projection of $X$ on the first $k-1$ coordinates is equal to $Y$ (where $X$ is $k$-ary);

4. the cartesian product of $X$ and $Y$ is equal to $Z$;

5. the intersection of $X$ and $Y$ is equal to $Z$;

6. $X$ is equal to the group $Y$ up to a fixed permutation of coordinates;

7. the index of $X$ in $Y$ is equal to $k$;

8. the index of $X$ in $Y$ is greater or equal to $k$;

9. the tuple $c$ is in $X$;

Remark that the sentences of types $3,4,5$ and 6 correspond to the equivalence sentences which occur in $\mathbb{A}$.

Note that every subgroup $G_{0}$ of $G$ which contains all the constants, satisfies the axioms of types $2,4,5,6$, and 9 . For each sentence $\Psi_{X, Y, k}$ of types 7 or 8 , a subgroup $G_{0}$ satisfies $\Psi_{X, Y, k}$ if and only if it contains at least $k$ elements of $Y$ which are in different cosets modulo $X$. Thus there exist finitely generated subgroups of $G$ which satisfy the finite set of axioms of types $1,2,4,5,6,7,8$ and 9 .

To deal with axioms of type 3 , we need to find finitely generated subgroups which are also "closed under projection" in the adequate sense. This is done in the two following claims. We say that a subset $X$ of $G$ is stable under quasi-endomorphisms if for each $x \in X$ and each $\alpha \in \mathbb{F}_{q}$, the set $\left\{y \in G:(x, y) \in H_{\alpha}\right\}$ is a subset of $X$.

Claim. Let $X$ be a definable subgroup of $G^{k}$. Then there exists a finite subset $D_{X}$ of $G$ such that, if $G_{0}$ is any subgroup stable under quasi-endomorphisms which contains $D_{X}$, if $\pi$ denotes the projection from $G^{k}$ onto the first $k-1$ coordinates, then $\pi(X) \cap G_{0}^{k-1}=$ $\pi\left(X \cap G_{0}^{k}\right)$.

Proof. Let $l$ be the dimension (algebraic dimension $=$ Morley rank) of $X$ and $\left(a_{1}, \ldots, a_{k}\right)$ a generic point of $X$, that is a point of dimension $l$. Then, there are two cases.

Either, $a_{k}$ is independent of $a_{1}, \ldots, a_{k-1}$. It follows easily in this case that $X=Y \times G$ where $Y=\left\{\left(x_{1}, \ldots, x_{k-1}\right):\left(x_{1}, \ldots, x_{k-1}, 0\right) \in X\right\}$ and then for every subgroup $G_{0}$, $\pi(X) \cap G_{0}^{k-1}=\pi\left(X \cap G_{0}^{k}\right)$. 
Otherwise, by a permutation of coordinates we can assume that $a_{l+1}, \ldots, a_{k}$ are algebraic over $a_{1}, \ldots, a_{l}$. (Note that then every generic of $X$ satisfies this property.) For each $j, l<j \leq k, a_{j} \in \operatorname{acl}\left(a_{1}, \ldots, a_{l}\right)$; so (see Fact 2.1), there exist $\alpha_{j, 1}, \ldots, \alpha_{j, l} \in \mathbb{F}_{q}$ and $b_{j, 1}, \ldots, b_{j, l} \in G$ such that $a_{j}^{\prime}=a_{j}-\sum_{1 \leq i \leq l} b_{j, i} \in \operatorname{acl}(\emptyset)$ and for every $i \leq l$, $\left(a_{i}, b_{j, i}\right) \in H_{\alpha_{j, i}}$.

Let $T$ be the subgroup of $G^{k}$ of elements $\left(x_{1}, \ldots, x_{k}\right)$ such that there exist $y_{l+1}, \ldots, y_{k}$ with $\left(x_{1}, \ldots, x_{l}, y_{l+1}, \ldots, y_{k}\right) \in X$ and for each $j, l<j \leq k$, there exist $y_{j, 1}, \ldots, y_{j, l}$ with $x_{j}=y_{j}-\sum_{i} y_{j, i}$ and $i \leq l,\left(x_{i}, y_{j, i}\right) \in H_{\alpha_{j, i}}$. Then $\left(a_{1}, \ldots, a_{l}, a_{l+1}^{\prime}, \ldots, a_{k}^{\prime}\right) \in T$. Now let $T^{\prime}:=\left\{\left(x_{l+1}, \ldots, x_{k}\right):\left(0, \ldots, 0, x_{l+1}, \ldots, x_{k}\right) \in T\right\}$.

We claim that $T^{\prime}$ is finite and that $T=G^{l} \times T^{\prime}$. Since $X$ is of dimension $l$, the group $X^{\prime}:=\left\{\left(x_{l+1}, \ldots, x_{k}\right):\left(0, \ldots, 0, x_{l+1}, \ldots, x_{k}\right) \in X\right\}$ is finite. It follows that $T^{\prime}$ is finite because the cokernels of the quasi-endomorphisms are finite. Let $\left(x_{1}, \ldots, x_{l}\right)$ be a generic of $G^{l}$ over $\left(a_{1}, \ldots, a_{l}\right)$. By strong minimality, $\left(x_{1}, \ldots, x_{l}\right)$ and $\left(a_{1}, \ldots, a_{l}\right)$ have the same type over $\operatorname{acl}(\emptyset)$. Since $\left(a_{l+1}^{\prime}, \ldots, a_{k}^{\prime}\right) \in \operatorname{acl}(\emptyset)$, we have $\left(x_{1}, \ldots, x_{l}, a_{l+1}^{\prime}, \ldots, a_{k}^{\prime}\right) \in T$ and so $\left(x_{1}-a_{1}, \ldots, x_{l}-a_{l}, 0, \ldots, 0\right) \in T$. But $\left(x_{1}-a_{1}, \ldots, x_{l}-a_{l}\right)$ is generic, so $G^{l} \times\{0\}^{k-l} \subset T$ and thus $T=G^{l} \times T^{\prime}$.

Now, let $G_{0}$ be any subgroup of $G$ stable under quasi-endomorphisms such that $G_{0}^{k-l}$ contains $T^{\prime}$. Then $\pi(X) \cap G_{0}^{k-1}=\pi\left(X \cap G_{0}^{k}\right)$ : indeed let $\left(x_{1}, \ldots, x_{l}, y_{l+1}, \ldots, y_{k}\right) \in X$ be such that $x_{1}, \ldots, x_{l} \in G_{0}$, we are going to show that $y_{k} \in G_{0}$. For each $j, l<j \leq k$, take $y_{j, 1}, \ldots, y_{j, l}$ such that for each $i \leq l,\left(x_{i}, y_{j, i}\right) \in H_{\alpha_{j, i}}$. Then, by stability of $G_{0}$ under quasi-endomorphisms, $y_{j, i} \in G_{0}$ for each $j, l<j \leq k$, and each $i \leq l$. For each $j$, $l<j \leq k$, let $z_{j}=y_{j, 1}+\ldots+y_{j, l}$ and $x_{j}=y_{j}-z_{j}$. Then $\left(x_{1}, \ldots, x_{l}, x_{l+1}, \ldots, x_{k}\right) \in T$ and as $T=G^{l} \times T^{\prime},\left(x_{l+1}, \ldots, x_{k}\right) \in T^{\prime} \subset G_{0}^{k-l}$. So, in particular, $x_{k} \in G_{0}$ and thus $y_{k}=x_{k}+z_{k} \in G_{0}$.

Claim. For every finite subset $A \subset G$, there is a finite subgroup $G_{0}$ of $G$, containing $A$, which is stable under quasi-endomorphisms.

Proof. Let $A$ be a finite subset of $G$. For a subset $X$ of $G$ denote by $\bar{X}$ the set $\cup_{(x, \alpha) \in X \times \mathbb{F}_{q}}\left\{y \in G:(x, y) \in H_{\alpha}\right\}$. Note that $\bar{X}$ is not necessarily stable under quasiendomorphisms (i.e. $\overline{\bar{X}}$ is not necessarily equal to $\bar{X}$ ).

For each $(\alpha, \beta) \in \mathbb{F}_{q}^{2}$, let $H_{\alpha} \circ H_{\beta}$ denote the subgroup of $G^{2}$ defined by the formula

$$
\exists z\left((x, z) \in H_{\beta} \wedge(z, y) \in H_{\alpha}\right) .
$$

The quasi-endomorphism $H_{\alpha \beta}$ is equal to the connected component of $H_{\alpha} \circ H_{\beta}$. Let $X_{0}:=\left\{y \in G:(0, y) \in H_{\alpha} \circ H_{\beta}\right.$ for some $\left.(\alpha, \beta) \in \mathbb{F}_{q}{ }^{2}\right\}$ and let $A_{0}$ be the finite subgroup generated by $A$ and $\overline{X_{0}}$. Let $B$ be the set $\overline{A_{0}}$. We prove that $B$ is stable under quasi-endomorphisms : let $\alpha \in \mathbb{F}_{q}, x \in B$ and $y \in G$ be such that $(x, y) \in H_{\alpha}$. By definition of $B$, there exists $\beta \in \mathbb{F}_{q}$ and $z \in A_{0}$ such that $(z, x) \in H_{\beta}$. So $(z, y) \in H_{\alpha} \circ H_{\beta}$. Let $y^{\prime} \in G$ be such that $\left(z, y^{\prime}\right) \in H_{\alpha \beta}$. Then $y-y^{\prime} \in X_{0}$ since $\left(0, y-y^{\prime}\right) \in H_{\alpha} \circ H_{\beta}$. Remark that if $\alpha=0$ then $y=0$ and if $\beta=0$ then $y \in X_{0}$. So assume that $\alpha \beta \neq 0$. Let $t \in G$ be such that $\left(y-y^{\prime}, t\right) \in H_{(\alpha \beta)^{-1}}$. Then $t \in A_{0}$ since $y-y^{\prime} \in X_{0}$ and $\overline{X_{0}} \subseteq A_{0}$. Thus $y \in B$ since $\left(z+t, y^{\prime}+\left(y-y^{\prime}\right)\right) \in H_{\alpha \beta}$ and $z+t \in A_{0}$. Now consider $G_{0}$ the subgroup generated by $B$. Then $G_{0}$ is also stable under quasi-endomorphisms since for 
each $x_{1}, x_{2} \in G$ and each $\alpha \in \mathbb{F}_{q}$,

$$
\left\{y \in G:\left(x_{1}+x_{2}, y\right) \in H_{\alpha}\right\}=\left\{y_{1} \in G:\left(x_{1}, y_{1}\right) \in H_{\alpha}\right\}+\left\{y_{2} \in G:\left(x_{2}, y_{2}\right) \in H_{\alpha}\right\} .
$$

Now by the previous claims we can find a finite subgroup of $G$, which contains sufficiently many elements in different cosets for the axioms of type 7 or 8 to be satisfied, which is stable under quasi-endomorphisms and contains each $D_{H_{i}}$. Such a finite group is a model of $\mathbb{T}$.

Corollary 3.17. $\mathbb{A}_{1}$ is a finite axiomatization of the complete theory $\mathbb{T}_{1}$.

Proof. By lemma 3.15, it suffices to show that every model of $\mathbb{A}_{1}$ is infinite. Let $\mathbb{S}$ be a model of $\mathbb{A}_{1}$. We work in the structure $\mathbb{G}_{S}$ which is a model of $\mathbb{T}$ by 3.14. For $r \in K$, let $\phi_{r}$ denote the corresponding $p p$-formula (over $\emptyset$ ). In $\mathbb{G}_{S}$, the kernel and cokernel of $\phi_{r}$ are finite, hence, by Lemma 3.14, they must be contained in $G \oplus\{0\}$ This means that $\phi_{r}$ restricted to $\{0\} \oplus S$ is a well-defined map. Let $s \in S \backslash\{0\}$. For each $r \in K$, consider the unique $\left(x_{r}, y_{r}\right) \in G_{S}$ such that $\left((0, s),\left(x_{r}, y_{r}\right)\right) \in \phi_{r}$. Then, if $r \neq r^{\prime}, y_{r} \neq y_{r^{\prime}}$ : indeed, if for $r \neq r^{\prime}, y_{r}=y_{r^{\prime}}$ then $\left((0, s),\left(x_{r}-x_{r^{\prime}}, 0\right)\right) \in\left(\phi_{r}-\phi_{r^{\prime}}\right)$, $\left(\left(x_{r}-x_{r^{\prime}}, 0\right),(0, s)\right) \in\left(\phi_{r}-\phi_{r^{\prime}}\right)^{-1}$ and hence $s=0$.

By Proposition 2.4, $\mathbb{T}_{1}$ is interdefinable with the theory of non trivial $K$-vector spaces, where $K$ is the ring of quasi-endomorphisms of $\mathbb{G}$. By Corollary 3.17 and Lemma 3.2, the theory of $K$-vector spaces is finitely axiomatizable. By Proposition 3.13 we derive immediately:

Corollary 3.18. The division ring $K$ of quasi-endomorphisms of $\mathbb{G}$ is finitely presented as a ring.

\subsection{Finitely axiomatizable strongly minimal groups}

Theorem 3.19. Let $\mathcal{G}$ be a strongly minimal group. If $T h(\mathcal{G})$ is finitely axiomatizable in a finite language $L$, then the quasi-endomorphisms ring of $\mathcal{G}$ is an infinite division ring which is finitely presented as a ring.

Proof. By [10], a finitely axiomatizable strongly minimal group must be locally modular, hence one-based.

Claim. If $\mathcal{G}$ is a strongly minimal locally modular group, and $\mathcal{G}^{\prime}$ is an expansion of $\mathcal{G}$ by a set $C$ of constants, then $\mathcal{G}$ and $\mathcal{G}^{\prime}$ have the same quasi-endomorphisms ring.

Proof. . For $A \subset G$, let $S$ be any connected $A$-definable subgroup of $G \times G$ in $\mathcal{G}^{\prime}$. Then $S$ is also definable in $\mathcal{G}$, over $A \cup C$. As $\mathcal{G}$ is one-based, by Fact 3.5, $S$ is definable over $\operatorname{acl}^{e q}(\emptyset)$ in $\mathcal{G}$. Conversely, any definable connected subgroup $H$ in $\mathcal{G}$ remains connected in $\mathcal{G}^{\prime}$. 
Recall the construction from Corollary 3.7: we add finitely many constants from $\mathcal{M}_{0}$, the prime model of $T h(\mathcal{G})$. Let $\mathcal{G}^{\prime}$ denote the expansion of $\mathcal{G}$ to the new language $L^{\prime}=L \cup\left\{m_{0}, \ldots, m_{n}\right\}$. Then $\mathcal{G}^{\prime}$ is interdefinable with some finitely axiomatizable abelian structure $\mathbb{G}=<G,+,-, 0, H_{1}, \ldots, H_{k}, m_{0}, \ldots, m_{n}>$. It follows that $\mathbb{G}$ and $\mathcal{G}$ have the same quasi-endomorphisms ring, as a quasi-endomorphism is a definable connected subgroup of $G \times G$. (Note that, as in $\mathbb{G}$ every definable connected subgroup is defined over $\emptyset$ (Proposition 1.6), the same is true in $\mathcal{G}^{\prime}$. Hence in $\mathcal{G}$ every definable connected subgroup was already definable over $\left\{m_{0}, \ldots, m_{n}\right\}$.)

By Corollary 3.18, the division ring of quasi-endomorphisms of $\mathbb{G}$ is finitely presented as a ring. As remarked above, this is also the division ring of quasi-endomorphisms of $\mathcal{G}$.

By Corollary 3.12, we derive immediately:

Corollary 3.20. Let $\mathcal{G}$ be a Morley Rank one group. If $\mathcal{G}$ is finitely axiomatizable then the quasi-endomorphism ring of its connected component is an infinite division ring which is finitely presented as a ring

\section{References}

[1] A. I. Abakumov, E. A. Paljutin, M. A. Tă̌clin, and Ju. E. Šišmarev. Categorial quasivarieties. Algebra i Logika, 11:3-38, 121, 1972.

[2] Gisela Ahlbrandt and Martin Ziegler. Quasi-finitely axiomatizable totally categorical theories. Ann. Pure Appl. Logic, 30(1):63-82, 1986. Stability in model theory (Trento, 1984).

[3] Walter Baur. Elimination of quantifiers for modules. Israel J. Math., 25(1-2):64-70, 1976.

[4] Steven Buechler. Essential stability theory. Perspectives in Mathematical Logic. Springer-Verlag, Berlin, 1996.

[5] Steven Buechler. Vaught's conjecture for superstable theories of finite rank. preprint, February 2006.

[6] G. Cherlin, L. Harrington, and A. H. Lachlan. $\aleph_{0}$-categorical, $\aleph_{0}$-stable structures. Ann. Pure Appl. Logic, 28(2):103-135, 1985.

[7] E. Fisher. Abelian structures. Ph.D. Thesis, 1975.

[8] Hans B. Gute and K. K. Reuter. The last word on elimination of quantifiers in modules. J. Symbolic Logic, 55(2):670-673, 1990.

[9] Wilfrid Hodges. Model theory, volume 42 of Encyclopedia of Mathematics and its Applications. Cambridge University Press, Cambridge, 1993. 
[10] Ehud Hrushovski. Finitely axiomatizable $\aleph_{1}$-categorical theories. J. Symbolic Logic, 59(3):838-844, 1994.

[11] Ehud. Hrushovski and Anand Pillay. Weakly normal groups. In Logic colloquium '85 (Orsay, 1985), volume 122 of Stud. Logic Found. Math., pages 233-244. NorthHolland, Amsterdam, 1987.

[12] A. A. Ivanov. The problem of finite axiomatizability for strongly minimal theories of graphs. Algebra i Logika, 28(3):280-297, 366, 1989.

[13] A. A. Ivanov. Strongly minimal structures with disintegrated algebraic closure and structures of bounded valency. In M. Weese and H. Wolter, editors, Proceedings of the Tenth Easter Conference on Model Theory, 1993.

[14] J. A. Makowsky. On some conjectures connected with complete sentences. Fund. Math., 81:193-202, 1974. Collection of articles dedicated to Andrzej Mostowski on the occasion of his sixtieth birthday, III.

[15] David Marker. Strongly minimal sets and geometry (tutorial). In J. A. Makowsky, editor, Proceedings Logic Colloquium '95, volume 11 of Lecture Notes in Logic, pages 191-213. Springer, Berlin, 1998.

[16] Michael Morley. Categoricity in power. Trans. Amer. Math. Soc., 114:514-538, 1965.

[17] E. A. Paljutin. Description of categorical quasivarieties. Algebra i Logika, 14(2):145185, 240, 1975.

[18] M. G. Peretjat'kin. Example of an $\omega_{1}$-categorical complete finitely axiomatizable theory. Algebra i Logika, 19(3):314-347, 382-383, 1980.

[19] Anand Pillay. Geometric stability theory, volume 32 of Oxford Logic Guides. The Clarendon Press Oxford University Press, New York, 1996. , Oxford Science Publications.

[20] Mike Prest. Model theory and modules, volume 130 of London Mathematical Society Lecture Note Series. Cambridge University Press, Cambridge, 1988.

[21] R. L. Vaught. Models of complete theories. Bull. Amer. Math. Soc., 69:299-313, 1963.

[22] B. I. Zilber. Solution of the problem of finite axiomatizability for theories that are categorical in all infinite powers. In Investigations in theoretical programming, (Russian), pages 69-74. Kazakh. Gos. Univ., Alma-Ata, 1981.

[23] B. I. Zilber. Uncountably categorical theories, volume 117 of Translations of Mathematical Monographs. American Mathematical Society, Providence, 1993. 
Institut Camille Jordan, Université Claude Bernard Lyon 1, 43 boulevard du 11 novembre 1918, 69622 Villeurbanne Cedex, France.

E-mail : blossier@math.univ-lyon1.fr

CNRS - Université Paris-Sud Orsay, Département de Mathématiques, Bat. 425, 91405 Orsay Cedex, France.

E-mail : elisabeth.bouscaren@math.u-psud.fr 